\title{
L3:PHI.CMD.P13.02 Support for CILC L1 Milestone Using STAR-CCM+
}

Stuart Slattery (ORNL)

Will Gurecky (UT-Austin)

10/7/2016

Approved for public release. Distribution is unlimited.
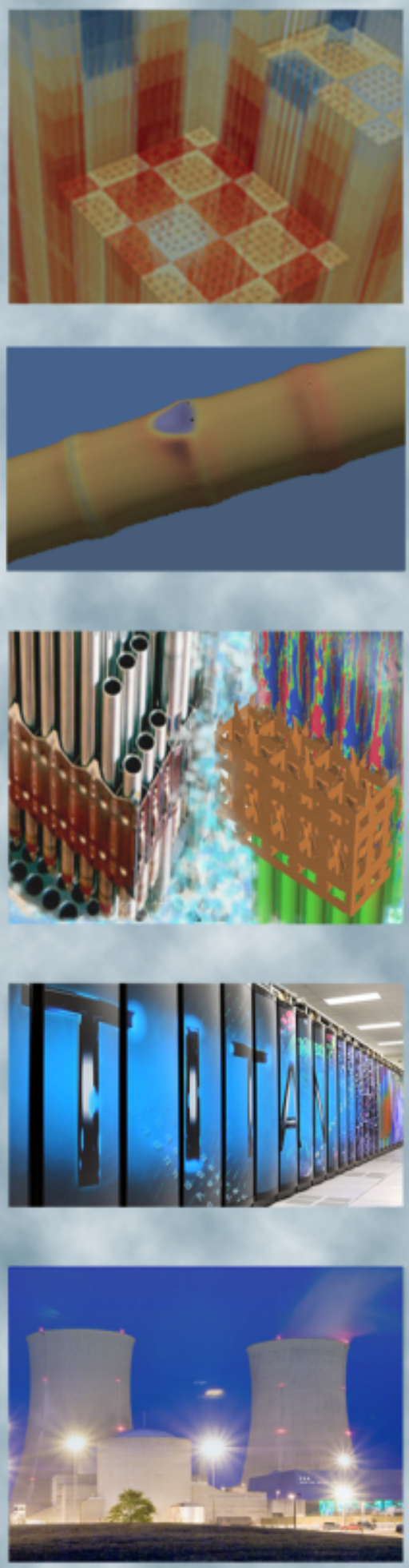


\section{REVISION LOG}

\begin{tabular}{|c|c|c|l|}
\hline Revision & Date & Affected Pages & \multicolumn{1}{c|}{ Revision Description } \\
\hline 0 & $10 / 7 / 2016$ & All & First Draft \\
\hline & & & \\
\hline & & & \\
\hline & & & \\
\hline
\end{tabular}

\section{Document pages that are:}

Export Controlled none

IP/Proprietary/NDA Controlled none

Sensitive Controlled none

\section{Requested Distribution:}

To: Jess Gehin, Dave Kropaczek, Kevin Clarno, Tom Downar, Jeff Banta

Copy: Stuart Slattery, Will Gurecky, Erich Schnieder

This report was prepared as an account of work sponsored by an agency of the United States Government. Neither the United States Government nor any agency thereof, nor any of their employees, makes any warranty, express or implied, or assumes any legal liability or responsibility for the accuracy, completeness, or usefulness of any information, apparatus, product, or process disclosed, or represents that its use would not infringe privately owned rights. Reference herein to any specific commercial product, process, or service by trade name, trademark, manufacturer, or otherwise, does not necessarily constitute or imply its endorsement, recommendation, or favoring by the United States Government or any agency thereof. The views and opinions of authors expressed herein do not necessarily state or reflect those of the United States Government or any agency thereof. 



\section{EXECUTIVE SUMMARY}

This report documents work performed to support CASL modeling of CIPS and CILC using the Cicada package. The work documented here is intended to complement current and future CIPS and CILC modeling activities in CASL. We provide tools for crud and corrosion related simulation and analysis by developing a better understanding of the interplay between the coupled physics that describe the phenomena at different time and length scales. We intend to use these models to better inform future simulation capability and development. This milestone has the following objectives:

1. Develop tools for the comparison of STAR-CCM+ and CTF to facilitate understanding model discrepancies

2. Develop tools and methods using STAR-CCM+ simulations to inform CTF simulations as a means of improving CIPS and CILC results at subchannel length scales

3. Develop a tool to assess the coupling of cladding heat transfer, oxidation, and crud growth for high fidelity CIPS and CILC modeling at CFD length scales

4. Quantify the effects of $1 \mathrm{D}$ vs. 3D coupled CIPS and CILC physics at CFD length scales

5. Develop an initial framework that could be used to demonstrate coupling of STAR-CCM+ to MAMBA 3D or other 3D crud models and the Bison fuel performance code for full 3D modeling of CIPS and CILC phenomena

We document the development of a new Cicada input format based on XML which facilitates more complex simulations and analysis along with a new HDF5 capability. This HDF5 capability allows for the reading and writing of STAR-CCM+ data directly to and from a binary file format which may be then passed to a suite of post-processing tools developed for the comparison of STAR-CCM+ with CTF as well as for statistical analysis. The statistical analysis forms the basis of STAR-CCM+ informed CTF calculations aimed at improving the coupling of CTF to crud and oxidation models. In addition, the new HDF5 capability and post-processing tools allow for analysis of CFD results within VERAView. Compatibility with VERAView, combined with restructuring Cicada, has greatly increased the integration of Cicada with VERA.

We then develop a new set of rudimentary multiphysics models of the cladding for CIPS and CILC analysis consisting of crud, corrosion, and thermal models to assess the coupling at CFD length scales. The new models allow for the comparison of 1D and 3D clad models and serve as a basis for future coupling with MAMBA 3D and potentially Bison using the DataTransferKit (DTK) library as the coupling tool. The results demonstrate the use of DTK for coupling STAR$\mathrm{CCM}+$ to an arbitrary computational mesh representing the fuel cladding to drive the coupled model. In the 1D and 3D comparisons, it was found that for fixed boundary conditions from CFD and neutronics, the coupled crud, corrosion, and thermal models yield essentially the same results for thin oxide layers while thicker oxidation creates stronger 3D effects. In addition, the 3D model varies substantially from the 1D model in terms of the heat flux used to couple with the CFD code and therefore further investigations are warranted before a choice of $1 \mathrm{D}$ vs. 3D can be made. 


\section{CONTENTS}

EXECUTIVE SUMMARY iii

CONTENTS

FIGURES vi

TABLES vii

ACRONYMS viii

1 INTRODUCTION 1

2 STAR-CCM+/CTF TOOL DEVELOPMENT 2

2.1 Cicada's Input Format . . . . . . . . . . . . . . . . . . . 2

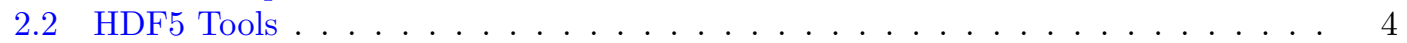

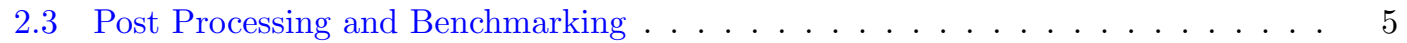

2.4 Future Work . . . . . . . . . . . . . . . . . . . . . . 8

3 COUPLED ALGORITHM ANALYSIS $\quad 10$

3.1 Coupled System . . . . . . . . . . . . . . . . . . . . . . 10

3.2 Crud Model . . . . . . . . . . . . . . . . . . . . . . . . . . . 11

3.3 Corrosion Model . . . . . . . . . . . . . . . . . . . . . . . . . 12

3.4 1D Thermal Model . . . . . . . . . . . . . . . . . . . . . . . . . . 13

3.5 3D Thermal Model . . . . . . . . . . . . . . . . . . . . . . . . 14

3.5.1 Finite Volume Discretization . . . . . . . . . . . . . . . . 14

3.5 .2 Cladding Consumption . . . . . . . . . . . . . . . 18

3.5 .3 Oxide-Metal Interface Temperature Evaluation . . . . . . . . . . . . . 18

3.5.4 Surface Heat Flux Reconstruction ．.. . . . . . . . . . . . . . . 19

3.6 Computational Tool . . . . . . . . . . . . . . . . . . . . . . . 19

3.6.1 Boundary Condition Interface and STAR-CCM+ Coupling . . . . . . . . 20

3.6 .2 Crud Model Interface . . . . . . . . . . . . . . . . . . . . . . . 20

3.6.3 Corrosion Model Interface . . . . . . . . . . . . . . . . . . . . . . . . . 21

3.6 .4 Clad Model Interface . . . . . . . . . . . . . . . . . . . . . . . . 21

3.7 CFD Model for Analysis . . . . . . . . . . . . . . . . . . . . . 22

3.8 Time Integration . . . . . . . . . . . . . . . . . . . . . . . . . . . . . 22

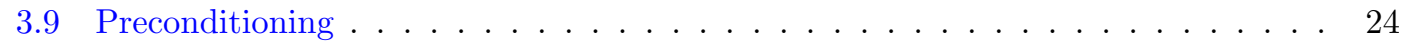

3.10 1D Mesh Refinement . . . . . . . . . . . . . . . . . . . . . 24

3.11 3D Mesh Refinement . . . . . . . . . . . . . . . . . . . 25

3.12 Model Comparison . . . . . . . . . . . . . . . . . . . . . . . 27

3.13 Model Comparison for Thick Oxide Layers . . . . . . . . . . . . . . . . . . 30

3.14 Algorithmic Assessment Summary . . . . . . . . . . . . . . . . . . . . . . . 31

4 CONCLUSION 33 


\section{FIGURES}

1 Power profile read from an external HDF5 file applied to the interior surface of the cladding. . . . . . . . . . . . . . . . . . . 3

2 Azimuthally binned surface temperature in subchannel $s 1$ on pin 1. Azimuthal segments are labeled $\{A s 1, \ldots A s 4\} \ldots \ldots \ldots \ldots \ldots$

3 Single pin CFD results visualized in VERAView. . . . . . . . . . . . 6

4 (a) Moderator - cladding interface temperature. (b) Bulk homogenized fluid temperature. (c) Turbulent kinetic energy at the moderator - cladding interface. (d) Axial velocity. . . . . . . . . . . . . . . . . . 7

5 Frequency binned field results tallied over a subset of the rod surface. Axial boundaries at $[259.7,267.0](\mathrm{cm})$. Results were generated at 160 percent nominal power in a single pin configuration. . . . . . . . . . . . . 9

6 Typical CFD computed rod surface temperature showing axial and azimuthal variation. . . . . . . . . . . . . . . . . . . . 14 


\section{TABLES}

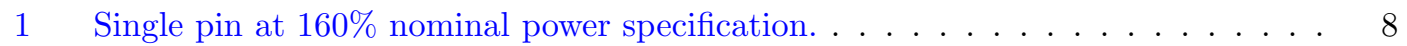

2 MAMBA1D model parameters. . . . . . . . . . . . . . . . . 11

$3 \quad$ EPRI/KWU/C-E corrosion model constants. . . . . . . . . . . . . . . . . 12

4 Single pin at $160 \%$ nominal power specification. . . . . . . . . . . . . 22

$5 \quad 1 \mathrm{D}$ model crud sub-cycling results. The crud model time step was fixed at 1.0e4 seconds while the clad model time step increases with sub-cycle count. . . . . . . 23

63 D model preconditioner reuse results with $\operatorname{ILUT}(1) \ldots \ldots \ldots \ldots$

7 1D model theta mesh refinement. Z cells fixed at 1000. . . . . . . . . . . . . 24

8 1D model $\mathrm{Z}$ mesh refinement. Theta cells fixed at 128. . . . . . . . . . . 25

9 1D model mesh refinement. Coarse (Theta $=32, \mathrm{Z}=250$ ) vs. fine (Theta $=128$,

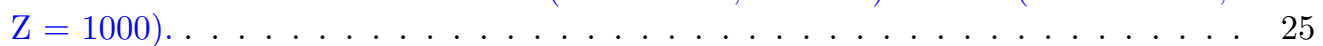

103 D model uniform radial mesh refinement. . . . . . . . . . . . . . . . 25

11 3D model radial mesh refinement with oxide layer. Oxide mesh is 25 microns thick with 0.5 micron mesh spacing. . . . . . . . . . . . . . . 26

12 3D model theta mesh refinement with oxide layer. Oxide mesh is 25 microns thick with 0.5 micron mesh spacing. $5 \mathrm{R}$ cells in remaining cladding and $250 \mathrm{Z}$ cells. . 26

13 3D model $\mathrm{Z}$ mesh refinement with oxide layer. Oxide mesh is 25 microns thick with 0.5 micron mesh spacing. $5 \mathrm{R}$ cells in remaining cladding and $32 \mathrm{Z}$ cells. . . 27

143 D vs. 1D model comparison at $100 \%$ power for 500 days. . . . . . . . . . . 28

$153 \mathrm{D}$ vs. $1 \mathrm{D}$ model comparison at $120 \%$ power for 500 days. . . . . . . . . . . . 28

163 D vs. 1D model comparison at $140 \%$ power for 500 days. . . . . . . . . . . . 29

17 3D vs. 1D model comparison at $160 \%$ power for 500 days. . . . . . . . . . . . 29

18 3D vs. 1D model comparison at $180 \%$ power for 500 days. . . . . . . . . . . . . 30

$193 \mathrm{D}$ vs. $1 \mathrm{D}$ model comparison at $180 \%$ power for 500 days with fast flux parameter of $6.0 \mathrm{e} 17 \mathrm{n} / \mathrm{cm}^{\wedge} 2$-s as defined by IAEA report. . . . . . . . . . . . . . 31

$203 \mathrm{D}$ vs. 1D model comparison at $160 \%$ power for 500 days with fast flux parameter of $6.0 \mathrm{e} 17 \mathrm{n} / \mathrm{cm}^{\wedge} 2$-s as defined by IAEA report. . . . . . . . . . . . . 31 


\section{ACRONYMS}

CASL Consortium for the Advanced Simulation of LWRs

CFD Computational Fluid Dynamics

CILC CRUD Induced Local Corrosion

CIPS CRUD Induced Power Shift

CRUD Chalk River Unidentified Deposit

CTF Cobra-Two Fluid (Cobra-TF)

MLE Maximum Likelihood Estimate

TH Thermal Hydraulic

VERA Virtual Environment for Reactor Applications 


\section{INTRODUCTION}

This report documents work performed to support CASL modeling of CIPS and CILC using the Cicada package. The work documented here is intended to complement current and future CIPS and CILC modeling activities in CASL and builds on previous developments in CASL as documented in $[2,4,3,8,10,9,5]$. We provide tools for crud and corrosion related simulation and analysis by developing a better understanding of the interplay between the coupled physics that describe the phenomena at different time and length scales. We intend to use these models to better inform future simulation capability and development. This milestone has the following objectives:

1. Develop tools for the comparison of STAR-CCM+ and CTF to facilitate understanding model discrepancies

2. Develop tools and methods using STAR-CCM+ simulations to inform CTF simulations as a means of improving CIPS and CILC results at subchannel length scales

3. Develop a tool to assess the coupling of cladding heat transfer, oxidation, and crud growth for high fidelity CIPS and CILC modeling at CFD length scales

4. Quantify the effects of $1 \mathrm{D}$ vs. 3D coupled CIPS and CILC physics at CFD length scales

5. Develop an initial framework that could be used to demonstrate coupling of STAR-CCM+ to MAMBA 3D or other 3D crud models and the Bison fuel performance code for full 3D modeling of CIPS and CILC phenomena

We document the development of a new Cicada input format based on XML which facilitates more complex simulations and analysis along with a new HDF5 capability. This HDF5 capability allows for the reading and writing of STAR-CCM+ data directly to and from a binary file format which may be then passed to a suite of post-processing tools developed for the comparison of STAR-CCM+ with CTF as well as for statistical analysis. The statistical analysis forms the basis of STAR-CCM+ informed CTF calculations aimed at improving the coupling of CTF to crud and oxidation models. In addition, the new HDF5 capability and post-processing tools allow for analysis of CFD results within VERAView. Compatibility with VERAView, combined with restructuring Cicada, has greatly increased the integration of Cicada with VERA.

We then develop a new set of rudimentary multiphysics models of the cladding for CIPS and CILC analysis consisting of crud, corrosion, and thermal models to assess the coupling at CFD length scales. The new models allow for the comparison of 1D and 3D clad models and serve as a basis for future coupling with MAMBA 3D and potentially Bison using the DataTransferKit (DTK) library as the coupling tool [7]. The results demonstrate the use of DTK for coupling STAR-CCM+ to an arbitrary computational mesh representing the fuel cladding to drive the coupled model. In the 1D and 3D comparisons, it was found that for fixed boundary conditions from CFD and neutronics, the coupled crud, corrosion, and thermal models yield essentially the same results for thin oxide layers while thicker oxidation creates stronger 3D effects. In addition, the 3D model varies substantially from the $1 \mathrm{D}$ model in terms of the heat flux used to couple with the CFD code and therefore further investigations are warranted before a choice of $1 \mathrm{D}$ vs. $3 \mathrm{D}$ can be made. 


\section{STAR-CCM+/CTF TOOL DEVELOPMENT}

Initially developed in pure C as a demonstrative tool for STAR-CCM+ and CRUD coupling, Cicada's capabilities have evolved to encompass a variety new technologies. Successful incorporation of the HDF5 library and portions of Trilinos have shown it is possible to leverage powerful $\mathrm{C} / \mathrm{C}++$ tools inside STAR-CCM+ user code. Cicada's I/O capabilities, usability and internal error handling benefited from the inclusion of these third party tools.

Significant steps have been taken to collect and distill CFD-scale CRUD and TH field data sets into useful formats. To this end, Cicada is accompanied by a powerful post processing pipeline which simplifies CTF-CFD comparison, statistical model inference, and interoperability with VERAView, a CASL visualization tool. The core data assimilation capabilities rely heavily on the HDF5 library. As a result of recent developments, Cicada is capable of exporting CFD field-data and finely resolved MAMBA results directly from a STAR-CCM+ simulation to the HDF5 format for later post processing.

An HDF5 read capability was implemented in Cicada to allow externally generated power profiles to be applied as a thermal boundary condition in the STAR-CCM+ domain. This feature enables loose out of memory coupling with a neutronics package.

\subsection{Cicada's Input Format}

Cicada's input file format has transitioned from a loosely formatted ASCII based input to a structured XML format. The XML is readily parsed into nested key-value pairs by the Teuchos subpackage in the Trilinos third-party library. Additional development flexibility and improved user-facing error messages have been realized as a result.

An example input is provided below in Listing (2). The input is representative of a typical single rod Cicada executed in a conjugate heat transfer mode. In this case an external power profile is extracted from an HDF5 file produced by MPACT and applied to the interior surface of the cladding as a heat flux boundary condition.

Cicada's capabilities can be toggled in the "global_inputs" ParameterList. This allows Cicada to be utilized as an HDF5 I/O bridge between an externally executed package without performing CRUD calculations, if desired. The MAMBA capability flag is specified as an integer rather than as boolean. In this way MAMBA can be executed intermittently at every $N$ inner CFD fixed point iterations. Additionally, if one wishes to perform CRUD computations without feedback to the CFD solution MAMBA only needs to be executed after the CFD solution has converged. Residual threshold inputs are present in the "global_inputs" ParameterList to accommodate this scenario. The execution of MAMBA will be delayed until the energy and continuity residual thresholds are met. MAMBA can be executed at every inner CFD iteration if the user specifies $N=1$.

The "coupling_regions" ParameterList block given in Listing 1 is composed in a hierarchical format. Individual surface regions are provided as subsidiary entities within volumetric regions. This format mimics the hierarchical entity specification in STAR-CCM+. In the Cicada input, a volumetric region is opened by providing the volume region name as it appears in the CFD model. Following the volumetric or surface region name a special "volume_fields" or "surface_fields" ParameterList respectively, modulates which data fields are written to an HDF5 file for later post processing. A default set of TH fields are exported in a given volume or surface region if user input is not provided. A STAR-CCM+ error is raised if a requested field is unavailable in the region. This situation can arise if a user misspecified a field name or if the requested field data is unavailable; for instance if the velocity field is requested for output in a solid region. 


\section{Listing 1. Coupled regions.}

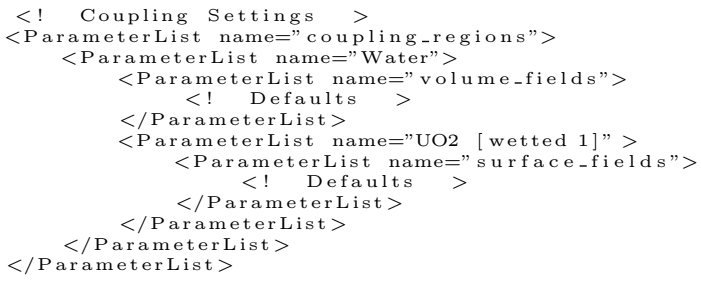

If "power_coupling" is enabled, an external HDF5 file containing pin powers in the standard VERA HDF5 format must be supplied. The total integrated power injected into the CFD domain must also be specified. A VERA HDF5 file can contain power profiles for many pins spanning the entire core. Therefore, in the "coupling_regions" ParameterList there exists an option to map a given pin's power profile to a CFD surface. Control over this mapping is enforced through an integer triplet: $\left\{\operatorname{pin}_{i k}, \operatorname{pin}_{j k}\right.$, assembly $\left._{k}\right\}$ where $\{i, j, k\}$ are indexed relative to zero. The input can accommodate multi-pin cases provided that each surface is uniquely named in the STAR model.

The axial power distribution is mapped to a CFD surface via 1D linear interpolation along the transverse axis. Power normalization is performed to obtain the correct heat flux magnitude such that the total energy injected into the domain is equivalent to the user specified value. Figure 1 displays a power profile obtained from MPACT applied on the interior surface of the cladding in STAR-CCM+.

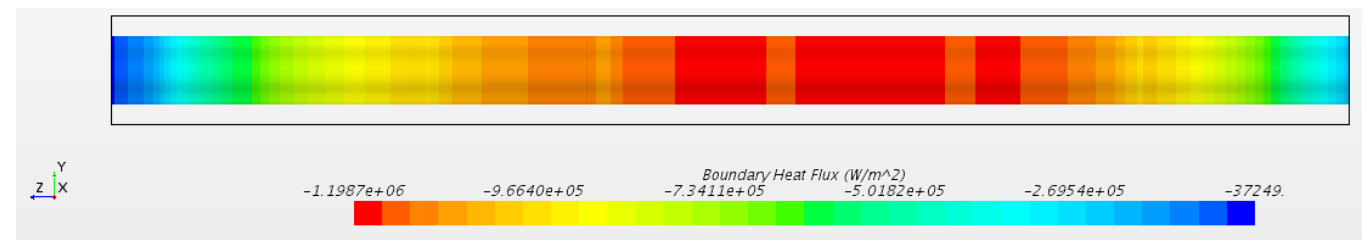

Figure 1. Power profile read from an external HDF5 file applied to the interior surface of the cladding. 


\section{Listing 2. Complete Cicada input example.}

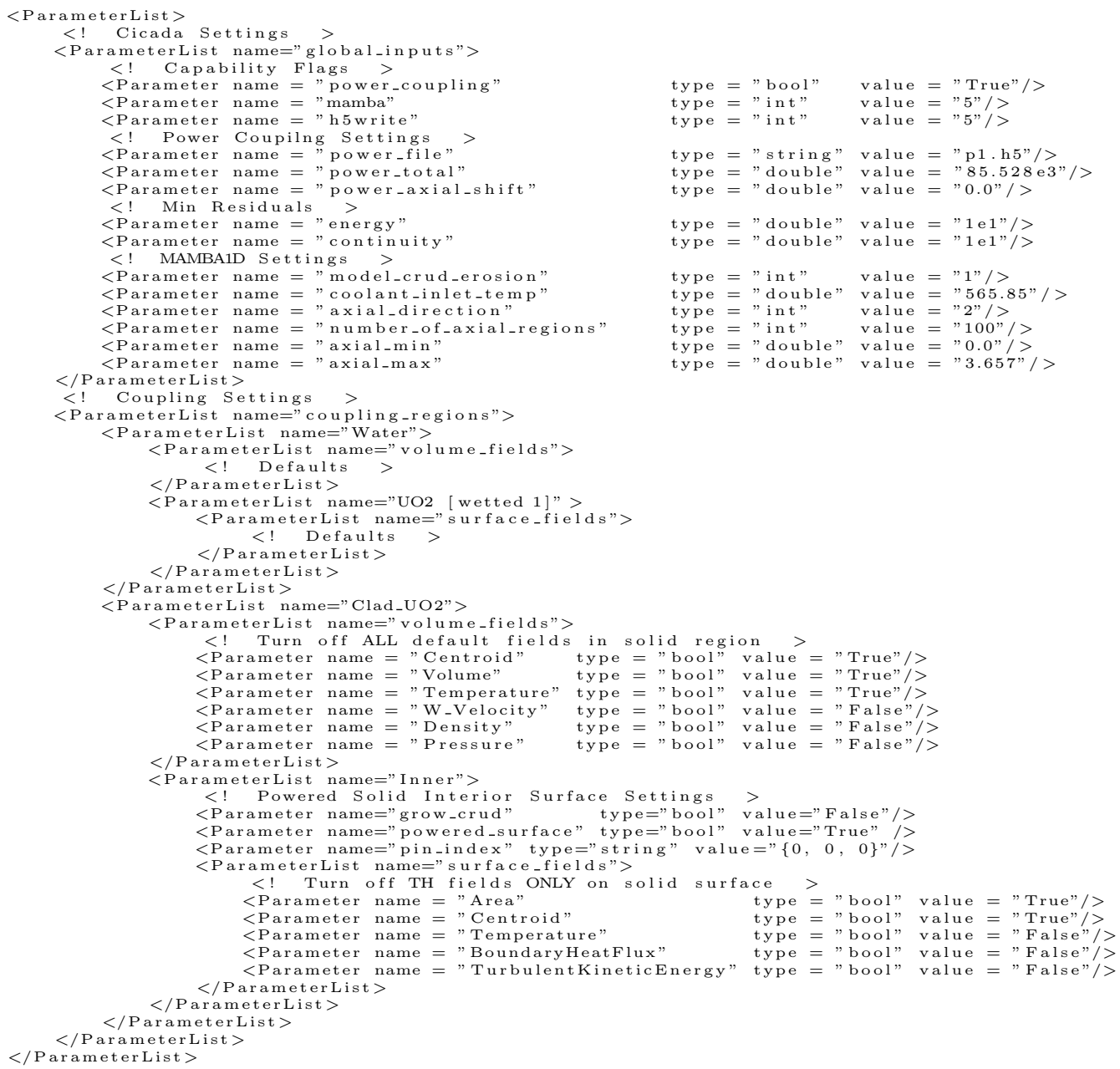

The XML form can accommodate new inputs when a new physics model is added to Cicada. A minimal amount of modification of the Cicada source code is needed to parse additional ParameterList entries. Additional code required to support new inputs predominately consists of bounds and type checking routines which provide detailed error output if an input parameter is improperly specified.

\subsection{HDF5 Tools}

The development of Cicada's HDF5 data export capabilities revealed weaknesses in the current tools provided by $\mathrm{CD}$-Adpaco for executing $\mathrm{C} / \mathrm{C}++$ user code inside STAR-CCM + . When writing I/O routines in user code it is desirable to leverage the parallel capabilities of the HDF5 library, however, STAR-CCM+ carries an internal dependency on a serial version (v1.8.6) of the HDF5 library. At the time of this writing, Cicada cannot be linked against a parallel version of the library due to symbol conflicts encountered between the user specified library compiled with parallel capabilities enabled and the serial-only hdf5 library built into STAR$\mathrm{CCM}+$. Collaboration with CD-Adapco targeted at addressing library conflicts could benefit future multiphysics coupling endeavours that leverage STAR's C-API.

STAR-CCM + is typically executed in a parallel environment in which each MPI processes owns a subset of the CFD domain with overlapping regions for solution transfer. Care must be observed that data from these overlapping "halo cells" are not included in the export. In a parallel environment field data is first collected on the root MPI process in sequential order before being exported to the HDF5 file. Consequently, the serial version of the HDF5 library is 
sufficient for performing data transfer. The sequential MPI send-receive paradigm guarantees that the ordering of data points is identical for each exported data field: E.g. the data is consistent with respect to global cell index in the output arrays.

The resultant HDF5 output from Cicada is written in a point wise format. For each user specified volumetric or surface region the respective cells' volume or area are exported alongside Cartesian coordinates of the cells' centroid. Likewise, thermal hydraulic and CRUD fields supported at the cells' centroids are written as desired. The raw CFD solution data residing in a Cicada HDF5 output file is useful for external multiphysics applications that are compatible with point-cloud data sets. Additionally, the availability of point cloud data in a high performance format facilitates data analysis outside of the STAR-CCM+ environment.

\subsection{Post Processing and Benchmarking}

Before performing a quantitative analysis of the difference between a STAR-CCM+ and CTF simulation, the point wise CFD data must first be distilled into a format compatible with the subchannel discretization CTF employs. The pointwise CFD field data must be integrated over subchannel-like parcels of space to accomplish this task.

Prior to performing the necessary integrals in the current implementation, the volumetric data is spatially binned by a series of boolean masking operations. Fortunately, the regular geometry of a PWR lattice simplifies the spatial binning procedure. A subchannel parcel can be constructed by placing a rectangular cuboid's vertices at the centroids of four neighbouring pin cells. Since a pin cell can be defined similarly, an abstract cuboid class provides the majority of binning logic. For a given cuboid interior, a series of three boolean masks, one for each cardinal axis, are applied to select only cells which reside inside the cuboid of interest. The masks are constructed by subjecting each CFD cell centroid to the distance conditions:

$$
\begin{array}{lll}
\left(x_{i}>=X 1\right) & \& \& & \left(x_{i}<X 2\right) \\
\left(y_{i}>=Y 1\right) & \& \& & \left(y_{i}<Y 2\right) \\
\left(z_{i}>=Z 1\right) & \& \& & \left(z_{i}<Z 2\right)
\end{array}
$$

Where $\{X 1, Y 1, Z 1, X 2, Y 2, Z 2\}$ are the bounding planes and $i$ is the CFD cell index. The boolean mask needs only to be computed once per subchannel and can be applied to all data sets residing in the region. Implementing a more efficient spatial binning algorithm may be required in order to post process assembly scale CFD results with $>50$ million cells. The current spatial binning routine has been applied to data sets with 2 million CFD cells yielding sub 30 second runtime.

The spatial binning routine can be applied recursively, further subdividing subchannel datasets into quarter-subchannel data sets, or azimuthally binned data sets sectioned about a pin centroid. Figure 2 shows four temperature traces extracted from a single pin-surface quadrant. This is representative of the default azimuthal resolution proved by MAMBA3D at 16 azimuthal bins per pin. The azimuthally binned surface temperature data is plotted against the transverse axis.

The resultant spatially binned and integrated CFD quantities are written to a format adhering to the standard VERA H5 file structure allowing for visualization of the CFD results in VERAView. A definition of the standard VERA H5 file structure is provided by PHI.VCS.P8.01 [http://www.casl.gov/docs/CASL-U-2014-0043-001.pdf]. Figure 3 depicts a visualization of single rod CFD results inside the VERAView software. The demonstrated compatibility with VERAView is an indication of the consistency that has been achieved between the post processed CFD data and the existing CTF HDF5 result format. Consequently, computing the difference between volume or area averaged CFD data and the equivalent CTF result is a trivial endeavour.

Comparisons between STAR-CCM + results with CTF provide can provide useful information to both CTF developers and CFD practitioners. A CFD-CTF comparison can serve to tune CTF grid loss and mixing coefficients for a given spacer grid design provided that a CFD simulation can explicitly define the spacer grid geometry, resolve mixing vane induced cross flow, local heat transfer coefficients, and pressure loss across the grid. 


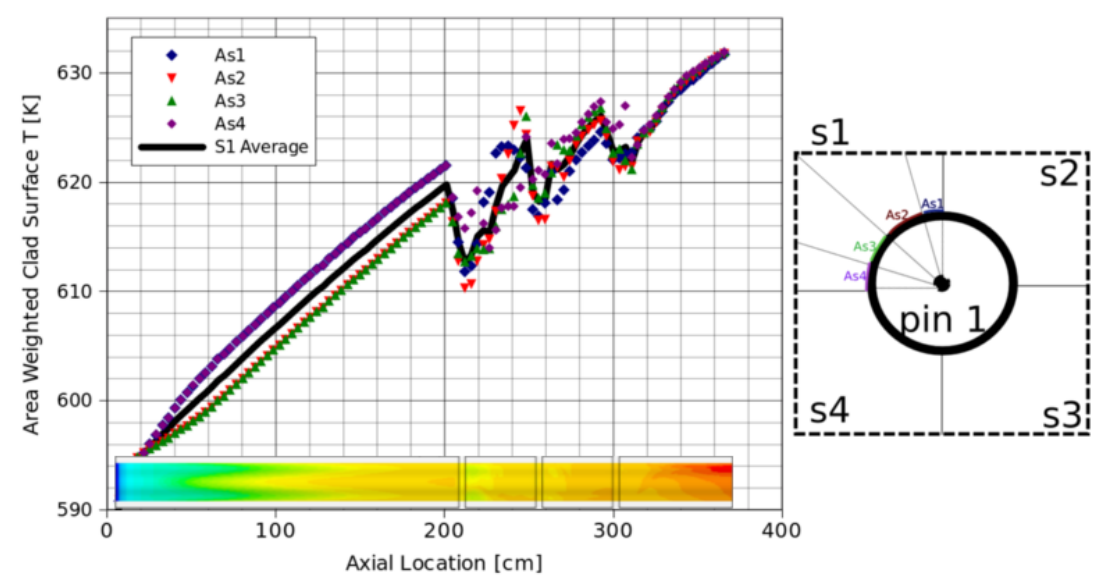

Figure 2. Azimuthally binned surface temperature in subchannel $s 1$ on pin 1. Azimuthal segments are labeled $\{A s 1, \ldots A s 4\}$.

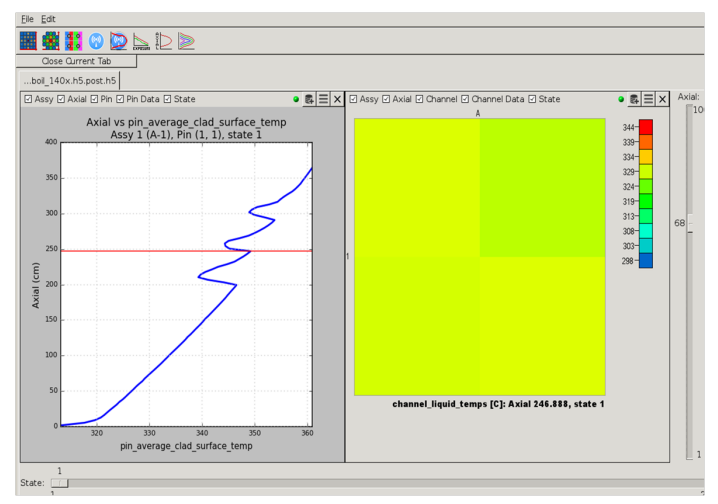

Figure 3. Single pin CFD results visualized in VERAView.

CFD practitioners can greatly benefit from comparison with CTF results in cases where two phase flow becomes important. Though STAR-CCM+ is contains fully segregated Eulerian two phase flow models and highly tunable subcooled boiling models, the sophistication brings little benefit if the parameters to these models are not tuned to reflect the flow regime at hand. CTF results provide one avenue for sanity checking the model parameters in the CFD two phase model; of course, a complete validation study of CFD boiling model must include experimental work. An example of such a two phase flow comparison is provided in Figure 4. STAR-CCM+'s Eulerian multiphase mixture model was selected to simplify the computation. The multiphase mixture model homogenizes the liquid and vapor phases into a single fluid. The Rohsenow wall boiling model was selected to compute the surface heat flux due to subcooled boiling and the vapor generation rate at the coolant-cladding interface.

The large surface temperature discrepancy is indicative of improper specification of boiling model parameters in the STAR-CCM+ model. Differences arising between the CFD and CTF bulk velocity and turbulent kinetic energy at the moderator-cladding interface reveal CTF does not account for the flow restriction of the spacer grid nor does CTF currently account for increased surface shear stresses due to increased turbulence downstream of a grid.

The single pin case was executed with the thermal hydraulic conditions given in Table 1. 


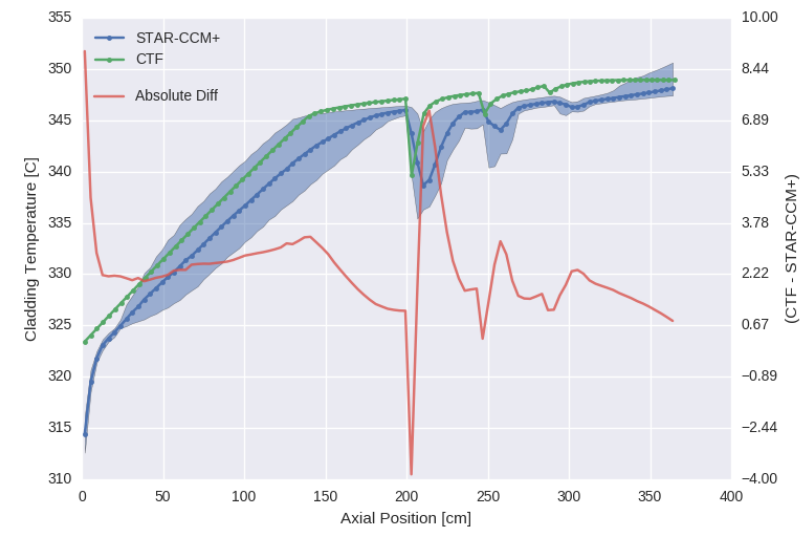

(a)

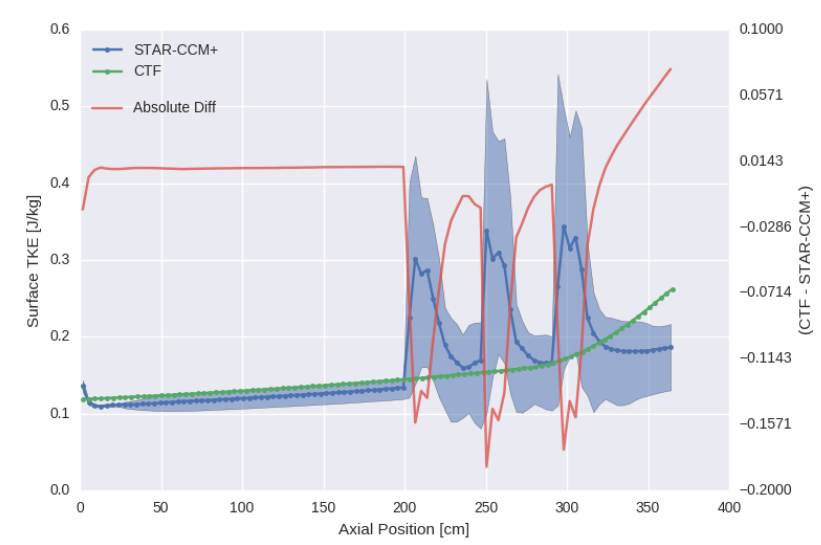

(c)

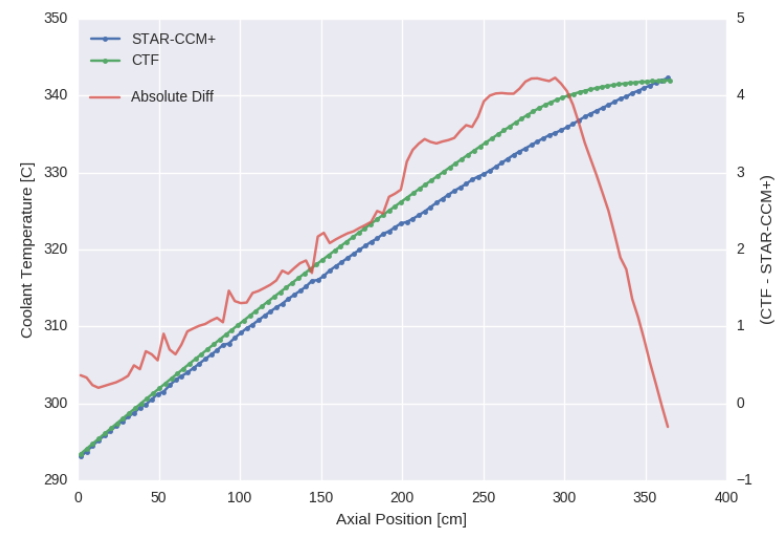

(b)

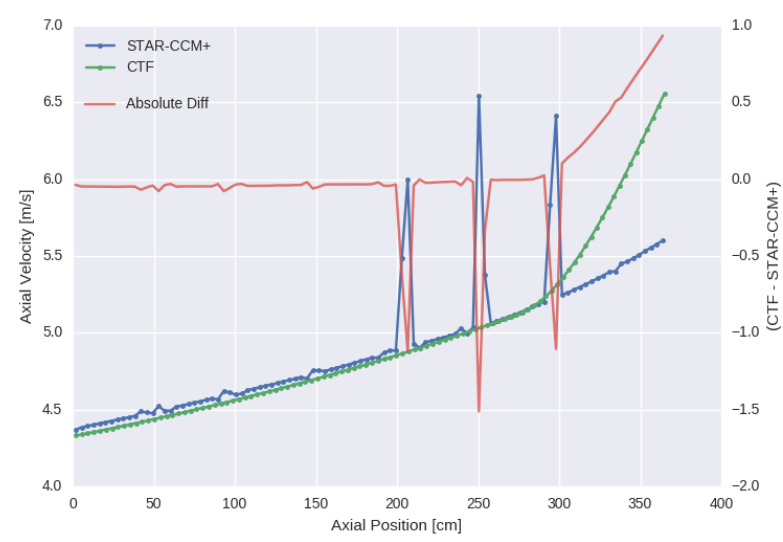

(d)

Figure 4. (a) Moderator - cladding interface temperature. (b) Bulk homogenized fluid temperature. (c) Turbulent kinetic energy at the moderator - cladding interface. (d) Axial velocity. 


\begin{tabular}{|l|c|}
\hline Rod height & $365.76[\mathrm{~cm}]$ \\
\hline Rod outer radius & $0.475[\mathrm{~cm}]$ \\
\hline Pin pitch & $1.6[\mathrm{~cm}]$ \\
\hline Inlet mass flow rate & $0.3[\mathrm{~kg} / \mathrm{s}]$ \\
\hline Inlet temperature & $565.85[\mathrm{~K}]$ \\
\hline Total integrated power (flat profile) & $107089[W]$ \\
\hline
\end{tabular}

Table 1. Single pin at $160 \%$ nominal power specification.

\subsection{Future Work}

Though Cicada is ideal for local hotspot and CRUD prediction within a single assembly it is not applicable to the full core scenario and is therefore not applicable to the CIPS problem outright. Core simulation with CFD scale TH resolution remains out of reach.

Figure 5 displays frequency binned CFD data tallied over a small section of a rod surface. CRUD growth favours areas of high surface temperature, low local shear stress (related to the local turbulent kinetic energy) and high heat flux. Furthermore, the B10 deposition model within MAMBA is sensitive to the local superheat. The relationship between boron hideout and surface temperature reveals a threshold behaviour with effectively zero boron hideout occurring in cells that reside below the saturation temperature. Thus it is not possible to accurately compute integral CRUD quantities without accounting for local variation in the TH solution around mixing vanes since there a strong potential to miss CRUD bound B10 deposits in these localized areas. CIPS prediction is a multi scale problem which requires the spatial fidelity of a CFD tool and the computational benefits of a nodal subchannel TH code to enable full core cycle depletion computations.

To bridge the scale gap, Cicada will play a central role by providing high fidelity training data sets upon which a bridging Hi2Low (high-to-low fidelity) model is constructed.

The construction of a Hi2Low model is conceptually similar to combining a nodal diffusion code calculation with a pre-computed high fidelity transport solution to resolve intra pin burnup and power distributions. One potential Hi2Low approach directly borrows from this analogy although it is not straightforward to preserve localized "outliers" - either hot or cold spots relative to the immediate surroundings - when mapping from the CFD scale to the sub-channel grid. Large training data set requirements and over fitting mitigation could present challenges when applying a pure CFD to CTF spatial interpolation procedure. Rather than attempting to predict the exact spatial distribution of temperature on a rod surface, it is perhaps more tractable to predict the fraction of the rod surface which exists above a given temperature. Future work will be targeted at identifying the likelihood of encountering hot or cold spots on a particular rod surface given a collection of boundary conditions, CFD data, and neighbouring $\mathrm{TH}$ conditions in the core. 

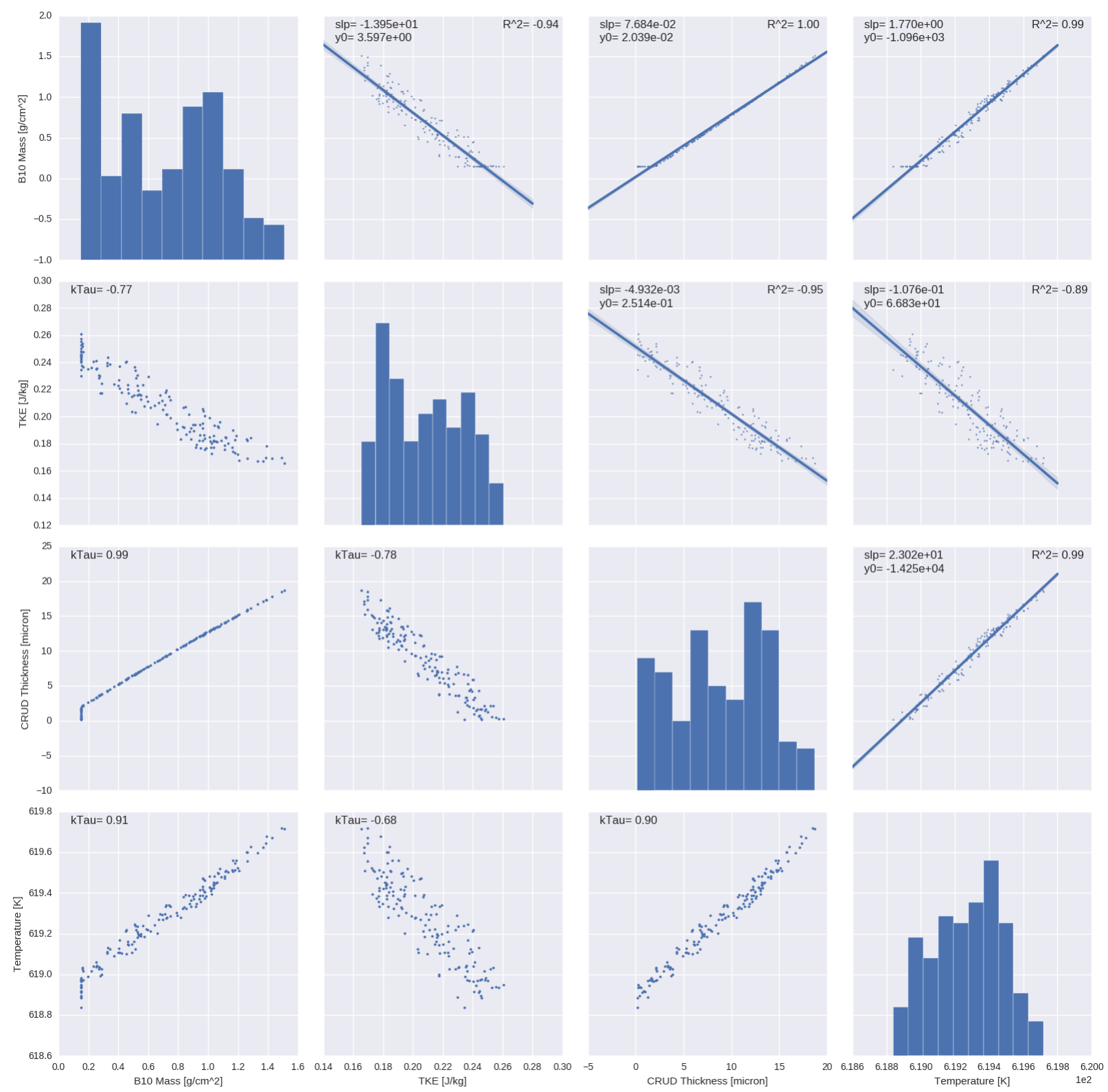

Figure 5. Frequency binned field results tallied over a subset of the rod surface. Axial boundaries at $[259.7,267.0](\mathrm{cm})$. Results were generated at $\mathbf{1 6 0}$ percent nominal power in a single pin configuration. 


\section{COUPLED ALGORITHM ANALYSIS}

In this section we will analyze the behavior of the coupled physics system used for the modeling of CILC, specifically, CFD, crud, and corrosion models. In addition, neutronics information is coupled in a weak sense where pin power profiles are provided as an energy source in the coupled system.

The coupled model is a rudimentary representation of the cladding using a thermal model, oxidation model, and crud model. This model is intended to be used in a conjugate heat transfer scheme with a CFD application. In conjugate heat transfer, given a heat flux output from the wetted cladding surface a new cladding surface temperature is computed by the CFD model:

$$
M_{c f d}\left(q_{\text {surf }}^{\prime \prime}\right)=T_{\text {surf }},
$$

and then using the new surface temperature the cladding model produces a new heat flux on the wetted surface of the clad:

$$
M_{\text {clad }}\left(T_{\text {surf }}\right)=q_{\text {surf }}^{\prime \prime},
$$

with these two models iterated until convergence is achieved.

In this work we will probe algorithmic choices for the clad model and assess the effects of these choices on the result of a single conjugate heat transfer iteration. Namely, given $T_{\text {surf }}$ from a CFD calculation, what is the resulting $q_{\text {surf }}^{\prime \prime}$ as a function of a given clad model and what is the resulting crud, oxidation, and thermal state of the clad? In this section we present the new coupling algorithm, describe the new computational capability developed to assess the coupling, and present results.

\subsection{Coupled System}

We divide the clad model into 3 physics models with input and output responses. The crud model computes the thermal resistance of the crud, $R_{\text {crud }}$ :

$$
M_{\text {crud }}\left(T_{\text {surf }}, q_{\text {surf }}^{\prime \prime}, \Delta t\right)=R_{\text {crud }},
$$

where $T_{\text {surf }}$ and $q_{\text {surf }}^{\prime \prime}$ are the wetted rod surface temperature and heat flux and $\Delta t$ is the time step over which to grow the crud. The corrosion model computes the current oxide thickness, $S:$

$$
M_{\text {corr }}\left(S_{0}, T_{\text {om }}, \Delta t\right)=S,
$$

where $S_{0}$ is the current oxide thickness, $\Delta t$ is the time step over which to grow the oxide layer, and is $T_{o m}$ the oxide/metal interface temperature which is reconstructed using the current temperature solution and the temperature at the crud/clad interface, $T_{c c}$. The crud/clad interface temperature is computed using the temperature drop across the crud layer and its thermal resistance:

$$
T_{c c}=T_{\text {surf }}+R_{\text {crud }} q_{\text {surf }}^{\prime \prime} .
$$

The thermal model solves the heat equation using thermal conductivities, $k$, computed using the current oxidized state of the cladding, to generate new clad temperatures:

$$
M_{\text {therm }}\left(T_{c c}, S, q_{\phi}^{\prime \prime}\right)=T,
$$

where $q_{\phi}^{\prime \prime}$ is the boundary heat flux on the inner surface of the cladding as generated by power from fission in a neutron transport calculation and the heat equation is defined as:

$$
\nabla \cdot k(S) \nabla T=0,
$$

with $k(S)$ indicating the dependence of the thermal conductivity on the thickness of the oxide layer. The resulting temperature solution can then be used to reconstruct the heat flux on the wetted surface of the clad. 
In general, the coupling of crud, oxidation, and thermal models is transient and nonlinear. For time integration, crud growth and oxidation occur on the time scales of hours and days while the cladding will thermally equilibrate on the time scale of seconds. Therefore, the cladding thermal model is assumed to be steady state. To resolve the nonlinearity, an operator split scheme is used in the time integration loop to evaluate the clad model. The clad model coupling algorithm is given in Algorithm 1. Note that this algorithm is intended to be executed at every conjugate heat transfer iteration as an evaluation of the clad model in Eq (2).

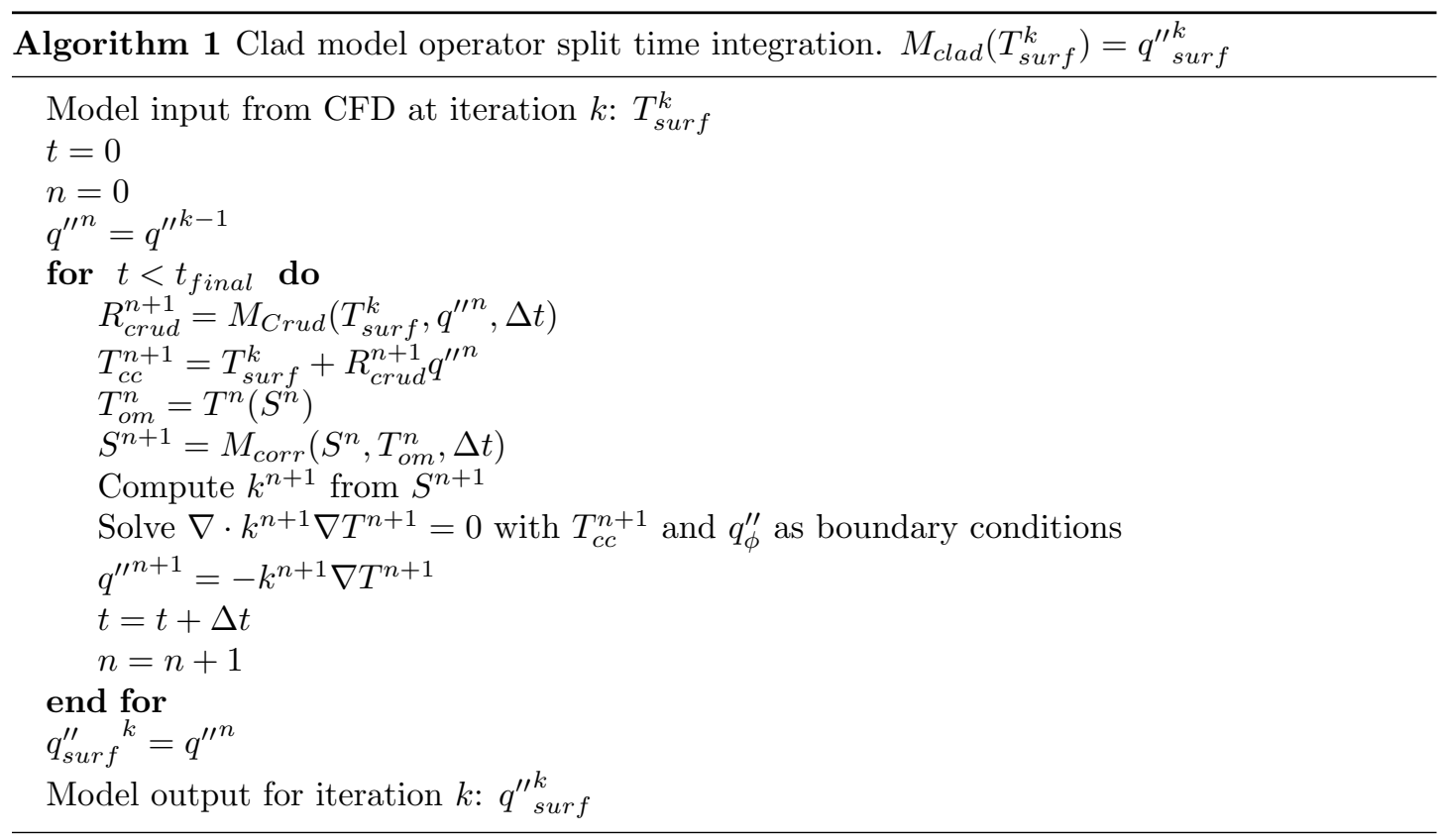

Within Algorithm 1, the crud, corrosion, and thermal models are not constrained to any specific formulation. Rather, different models may be used in this scheme. This includes MAMBA 1D or 3D for crud modeling, a variety of corrosion models, and 1D or 3D heat transfer. Next we describe the specific models used to obtain the results in this work.

\subsection{Crud Model}

MAMBA 1D was used as the crud model in this analysis. The following model parameters were used:

Table 2. MAMBA1D model parameters.

\begin{tabular}{ll}
\hline Parameter & Value \\
\hline Coolant Pressure & $1.55 \mathrm{e} 7 \mathrm{~Pa}$ \\
Coolant Dissolved Hydrogen & $3.2 \mathrm{e}-5 \mathrm{~m}^{\wedge} 3 / \mathrm{kg}$ \\
Coolant Boron Concentration & $1000 \mathrm{ppm}$ \\
Coolant Lithium Concentration & $3 \mathrm{ppm}$ \\
Coolant Soluble Nickel Concentration & $0.25 \mathrm{ppb}$ \\
Coolant Particulate Nickel Concentration & $2.2 \mathrm{ppb}$ \\
Coolant Soluble Iron Concentration & $2.2 \mathrm{ppb}$ \\
Coolant Inlet Temperature & $565.85 \mathrm{~K}$ \\
Time Step Size & $1 \mathrm{e} 4 \mathrm{~s}$ \\
\hline
\end{tabular}




\subsection{Corrosion Model}

Per the current CILC modeling in the L1 milestone, the EPRI/KWU/C-E corrosion model was used as described in the IAEA report on waterside corrosion of zirconium alloys [1]. In this model, two different rate equations for oxide growth are assumed, one for oxide thicknesses before the transition point and one for after the transition thickness. The pre-transition model is for oxide thickness $S$ is cubic:

$$
\frac{d S^{3}}{d t}=k_{1} \exp \left(Q_{1} /\left(R T_{o m}\right)\right)
$$

where $R, k_{1}$, and $Q_{1}$ are constants and $T_{o m}$ is the temperature at the oxide-metal interface. The post-transition model is:

$$
\frac{d S}{d t}=k_{2} \exp \left(Q_{1} /\left(R T_{o m}\right)\right)
$$

where again $R, k_{2}$, and $Q_{2}$ are constants and $T_{o m}$ is the temperature at the oxide-metal interface. The transition thickness is computed as:

$$
S_{\text {trans }}=D_{3} \exp \left(Q_{3} /\left(R T_{o m}\right)-E_{3} T_{o m}\right),
$$

with $S_{\text {trans }}$ the transition thickness and $D_{3}$ and $E_{3}$ constants. In Cicada, these rates are used to evaluate a new oxide thickness given the current oxide thickness, $S_{0}$, and a time step size, $\Delta t$. For pre-transition this is:

$$
S=\left[S_{0}{ }^{3}+\Delta t k_{1} \exp \left(Q_{1} /\left(R T_{o m}\right)\right)\right]^{1 / 3}
$$

and post-transition:

$$
S=S_{0}+\Delta t k_{2} \exp \left(Q_{1} /\left(R T_{\text {om }}\right)\right) .
$$

In the post-transition model, the $k_{2}$ coefficient is evaluated including an irradiation enhancement factor, $f$, such that $k_{2}$ is:

$$
k_{2}=C_{0} f
$$

where $C_{0}$ is a constant and $\mathrm{f}$ is:

$$
f=1+3.22(M \phi)^{P_{0}},
$$

with $M$ and $P_{0}$ constants and $\phi$ the rector fast flux.

For this work, the following constants were used:

Table 3. EPRI/KWU/C-E corrosion model constants.

\begin{tabular}{ll}
\hline Constant & Value \\
\hline$R$ & $1.987 \mathrm{cal} / \mathrm{mol}-\mathrm{K}$ \\
$k_{1}$ & $1.89 \mathrm{e} 10 \mathrm{microns} 3 /$ day \\
$Q_{1}$ & $32289 \mathrm{cal} / \mathrm{mol}$ \\
$Q_{2}$ & $27354 \mathrm{cal} / \mathrm{mol}$ \\
$D_{3}$ & $2.14 \mathrm{e} 7 \mathrm{microns}$ \\
$E_{3}$ & $1.17 \mathrm{e}-21 / \mathrm{K}$ \\
$C_{0}$ & $8.04 \mathrm{e} 7 \mathrm{microns} /$ day \\
$M$ & $7.46 \mathrm{e}-19 \mathrm{~cm}^{\wedge} 2-\mathrm{s} /$ neutron \\
$P_{0}$ & 0.24 \\
$\phi$ & $9.0 \mathrm{e} 14$ neutron $/ \mathrm{cm}^{\wedge} 2-\mathrm{s}$ \\
\hline
\end{tabular}

It should be noted that values of oxide thermal conductivity can vary widely as indicated by the IAEA report [1]. For this work a value of $1.5 \mathrm{~W} / \mathrm{m}-\mathrm{K}$ was used in all calculations. In 
addition, as indicated by Eq (13), the irradiation enhancement factor is applied to all posttransition oxidation. It should also be noted that the IAEA report uses a value of $6 \mathrm{e} 17$ for $\phi$ for many of its calculations. This seems rather large so for most calculations in this work a value of 9 e14 was used for $\phi$ as indicated in Table 3. However, this does significantly affect the resulting oxidation at high power and therefore we do include results later in the report using this value to show the difference.

\subsection{D Thermal Model}

A one-dimensional thermal model was developed with the intention of applying the model to each mesh cell face on the wetted rod interface. The model solves the heat conduction problem in the radial direction:

$$
q^{\prime \prime}=-k(r) \frac{d T}{d r} .
$$

A given radial segment of the mesh spans an azimuthal region, $\Delta \theta$, and an axial region, $\Delta z$. Given the flux boundary condition, $q_{\phi}^{\prime \prime}$, on the inner surface of the clad we define the total energy entering the radial zone as:

$$
Q=q_{\phi}^{\prime \prime} r_{i r} \Delta \theta \Delta z,
$$

where $r_{i r}$ is the inner radius of the clad. The total energy flow will be constant at any radial location in the zone leading to:

$$
\frac{Q}{\Delta \theta \Delta z}=-k(r) \frac{1}{r} \frac{d T}{d r},
$$

Assuming $k(r)$ is a constant, the solution to this differential equation is:

$$
T(r)=c_{1} \ln (r)+c_{2},
$$

with

$$
c_{1}=-\frac{Q}{k(r) \Delta \theta \Delta z},
$$

and

$$
c_{2}=T\left(r_{0}\right)-c_{1} \ln \left(r_{0}\right),
$$

where $T\left(r_{0}\right)$ is a known temperature at a known location $r_{0}$. The solution in the oxide region is found with constants:

$$
c_{1}^{\text {oxide }}=-\frac{Q}{k^{\text {oxide }} \Delta \theta \Delta z},
$$

and

$$
c_{2}^{\text {oxide }}=T_{c c}-c_{1}^{\text {oxide }} \ln \left(r_{\text {or }}\right),
$$

with $T_{c c}$ the crud/clad interface temperature and $r_{o r}$ the outer radius of the clad. The solution in the clad region is found with constants:

$$
c_{1}^{\text {clad }}=-\frac{Q}{k^{\text {clad }} \Delta \theta \Delta z},
$$

and

$$
c_{2}^{\text {clad }}=T_{o m}-c_{1}^{\text {clad }} \ln \left(T_{o r}-S\right),
$$

with $S$ the current oxide thickness and the oxide metal interface temperature computed as:

$$
T_{\text {om }}=c_{1}^{\text {oxide }} \ln \left(T_{\text {or }}-S\right)+c_{2}^{\text {oxide }} .
$$

Assuming the heat flux is very large, the maximum temperature in a radial zone is obtained by evaluating Eq (18) at the inner radius of the clad using the cladding material coefficients. For coupling back to the fluid model, the return heat flux is simply the energy flow through the outer clad surface or per Eq (16):

$$
q_{\text {surf }}^{\prime \prime}=q_{\phi}^{\prime \prime} \frac{r_{i r}}{r_{o r}} .
$$




\subsection{D Thermal Model}

The 3D thermal model provides a full dimensional representation of the cladding to better understand the effects of heat flow around the local thermal resistance provided by increased oxidation and crud growth at hot spots. These hot spots are expected due to the inclusion of spacer grids in the CFD model as shown in Figure 6 which introduces azimuthal variation in the surface temperature profile of the clad. The discretization of the heat equation in the cladding will be given followed by a derivation of quantities needed for coupling to the corrosion and crud models.

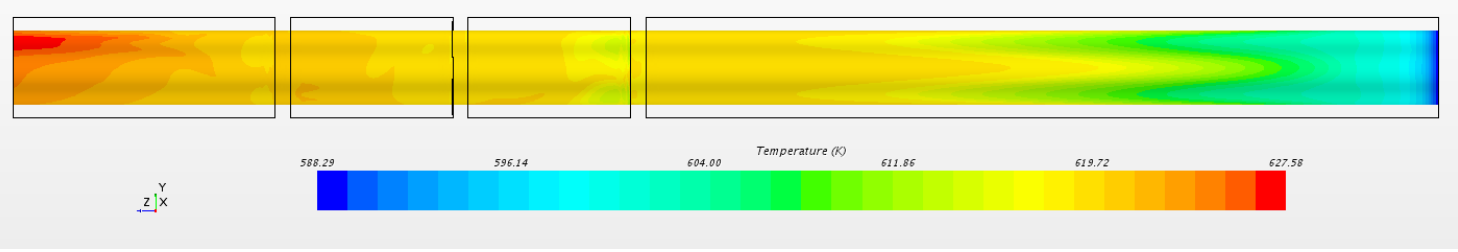

Figure 6. Typical CFD computed rod surface temperature showing axial and azimuthal variation.

\subsubsection{Finite Volume Discretization}

A finite volume scheme in cylindrical coordinates was used to discretize the heat equation in the cladding. We begin with the steady state heat equation:

$$
-\nabla \cdot k \nabla T=0
$$

or

$$
\nabla \cdot \mathbf{q}=0
$$

where the heat flux is defined as:

$$
\mathbf{q}=-k \nabla T .
$$

We integrate Eq (28) and apply the divergence theorem:

$$
\int_{\Omega} \nabla \cdot \mathbf{q} d \Omega=\int_{\Gamma} \mathbf{q} \cdot \mathbf{n} d \Gamma=0
$$

where $\Omega$ indicates an integration volume and $\Gamma$ indicates an integration surface with normal $\mathbf{n}$. To numerically calculate the integral we choose a control volume in cylindrical space that spans a radial region, $\Delta r$, an azimuthal region, $\Delta \theta$, and an axial region, $\Delta z$. For a given cell with cardinal index $i j k$ centered at $\left(r_{i}, \theta_{j}, z_{k}\right)$ with widths:

$$
\begin{aligned}
\Delta r_{i} & =r_{i+1 / 2}-r_{i-1 / 2} \\
\Delta \theta_{j} & =\theta_{j+1 / 2}-\theta_{j-1 / 2} \\
\Delta z_{k} & =z_{k+1 / 2}-z_{k-1 / 2}
\end{aligned}
$$

the integral is then decomposed into flux components orthogonal to each of the six faces of the cell, $f_{i j k}$, assuming a cell-constant temperature:

$$
\sum_{f_{i j k}}\left(\mathbf{q}_{f} \cdot \hat{\mathbf{n}}_{f}\right) A_{f}=0
$$

where $\mathbf{q}_{f}$ is the face-constant flux, $\hat{\mathbf{n}}_{f}$ is the face unit normal, $A_{f}$ is the surface area of the face, and it is assumed that the cell thermal conductivity, $k_{i j k}$ is constant. Satisfying Eq (32) for all cells in the computational mesh then amounts to the solution to the problem in Eq (27). 
We next define the cell face fluxes. The gradient in cylindrical coordinates is:

$$
\nabla=\left(\frac{\partial}{\partial r}, \frac{1}{r} \frac{\partial}{\partial \theta}, \frac{\partial}{\partial z}\right)
$$

which with Eq (29) gives:

$$
\mathbf{q}=-k \frac{\partial T}{\partial r}-k \frac{1}{r} \frac{\partial T}{\partial \theta}-k \frac{\partial T}{\partial z}
$$

For a cell centered at $\left(r_{i}, \theta_{j}, z_{k}\right)$ the face fluxes are:

$$
\begin{aligned}
q_{i+1 / 2} & =-k_{i+1 / 2} \frac{T_{i+1}-T_{i}}{\Delta r_{i+1 / 2}} \\
q_{i-1 / 2} & =-k_{i-1 / 2} \frac{T_{i}-T_{i-1 / 2}}{\Delta r_{i-1}} \\
q_{j+1 / 2} & =-k_{j+1 / 2} \frac{1}{r_{i}} \frac{T_{j+1}-T_{j}}{\Delta r_{j+1 / 2}} \\
q_{j-1 / 2} & =-k_{j-1 / 2} \frac{1}{r_{i}} \frac{T_{j}-T_{j-1}}{\Delta r_{j-1 / 2}} \\
q_{k+1 / 2} & =-k_{k+1 / 2} \frac{T_{k+1}-T_{k}}{\Delta r_{k+1 / 2}} \\
q_{k-1 / 2} & =-k_{k-1 / 2} \frac{T_{k}-T_{k-1 / 2}}{\Delta r_{k-1}},
\end{aligned}
$$

with:

$$
\begin{aligned}
\Delta r_{i+1 / 2} & =\frac{\Delta r_{i+1}+\Delta r_{i}}{2} \\
\Delta r_{i-1 / 2} & =\frac{\Delta r_{i}+\Delta r_{i-1}}{2} \\
\Delta \theta_{j+1 / 2} & =\frac{\Delta \theta_{j+1}+\Delta \theta_{j}}{2} \\
\Delta \theta_{j-1 / 2} & =\frac{\Delta \theta_{j}+\Delta \theta_{j-1}}{2} \\
\Delta z_{k+1 / 2} & =\frac{\Delta z_{k+1}+\Delta z_{k}}{2} \\
\Delta z_{k-1 / 2} & =\frac{\Delta z_{k}+\Delta z_{k-1}}{2}
\end{aligned}
$$

and $k_{i+1 / 2}$, for example, the effective thermal conductivity on the $i+1 / 2$ cell face.

Cell face conductivities are determined by first computing first-order representations of the 
face fluxes in a given cell, each of which can be computed in the current cell or the adjacent cell:

$$
\begin{aligned}
q_{i+1 / 2} & =-2 k_{i+1} \frac{T_{i+1}-T_{i+1 / 2}}{\Delta r_{i+1}} \\
q_{i+1 / 2} & =-2 k_{i} \frac{T_{i+1 / 2}-T_{i}}{\Delta r_{i}} \\
q_{i-1 / 2} & =-2 k_{i} \frac{T_{i}-T_{i-1 / 2}}{\Delta r_{i}} \\
q_{i-1 / 2} & =-2 k_{i-1} \frac{T_{i-1 / 2}-T_{i-1}}{\Delta r_{i-1}} \\
q_{j+1 / 2} & =-2 k_{j+1} \frac{1}{r_{i}} \frac{T_{j+1}-T_{j+1 / 2}}{\Delta \theta_{j+1}} \\
q_{j+1 / 2} & =-2 k_{j} \frac{1}{r_{i}} \frac{T_{j+1 / 2}-T_{j}}{\Delta \theta_{j}} \\
q_{j-1 / 2} & =-2 k_{j} \frac{1}{r_{i}} \frac{T_{j}-T_{j-1 / 2}}{\Delta \theta_{j}} \\
q_{j-1 / 2} & =-2 k_{j-1} \frac{1}{r_{i}} \frac{T_{j-1 / 2}-T_{j-1}}{\Delta \theta_{j-1}} \\
q_{k+1 / 2} & =-2 k_{k+1} \frac{T_{k+1}-T_{k+1 / 2}}{\Delta z_{k+1}} \\
q_{k-1 / 2} & =-2 k_{k} \frac{T_{k}-T_{k-1 / 2}}{\Delta z_{k}} \\
q_{k+1 / 2} & =-2 k_{k} \frac{T_{k+1 / 2}-T_{k}}{\Delta z_{k}} \\
\Delta z_{k-1} & T_{k-1}
\end{aligned}
$$

Equating each of these gives equations for the cell face temperatures:

$$
\begin{aligned}
T_{i+1 / 2} & =\frac{k_{i+1} \Delta r_{i} T_{i+1}+k_{i} \Delta r_{i+1} T_{i}}{k_{i+1} \Delta r_{i}+k_{i} \Delta r_{i+1}} \\
T_{i-1 / 2} & =\frac{k_{i} \Delta r_{i-1} T_{i}+k_{i-1} \Delta r_{i} T_{i-1}}{k_{i} \Delta r_{i-1}+k_{i-1} \Delta r_{i}} \\
T_{j+1 / 2} & =\frac{k_{j+1} \Delta \theta_{j} T_{j+1}+k_{j} \Delta \theta_{j+1} T_{j}}{k_{j+1} \Delta \theta_{j}+k_{j} \Delta \theta_{j+1}} \\
T_{j-1 / 2} & =\frac{k_{j} \Delta \theta_{j-1} T_{j}+k_{j-1} \Delta \theta_{j} T_{j-1}}{k_{j} \Delta \theta_{j-1}+k_{j-1} \Delta \theta_{j}} \\
T_{k+1 / 2} & =\frac{k_{k+1} \Delta z_{k} T_{k+1}+k_{k} \Delta z_{k+1} T_{k}}{k_{k+1} \Delta z_{k}+k_{k} \Delta z_{k+1}} \\
T_{k-1 / 2} & =\frac{k_{k} \Delta z_{k-1} T_{k}+k_{k-1} \Delta z_{k} T_{k-1}}{k_{k} \Delta z_{k-1}+k_{k-1} \Delta z_{k}} .
\end{aligned}
$$

Finally, the first order fluxes in Eq (37) are equated with the second order fluxes in Eq (35) and 
with the face temperatures in $\mathrm{Eq}(38)$ the following face thermal conductivities are obtained:

$$
\begin{aligned}
& k_{i+1 / 2}=\frac{\Delta r_{i+1}+\Delta r_{i}}{\frac{\Delta r_{i+1}}{k_{i+1}}+\frac{\Delta r_{i}}{k_{i}}} \\
& k_{i-1 / 2}=\frac{\Delta r_{i}+\Delta r_{i-1}}{\frac{\Delta r_{i}}{k_{i}}+\frac{\Delta r_{i-1}}{k_{i-1}}} \\
& k_{j+1 / 2}=\frac{\Delta \theta_{j+1}+\Delta \theta_{j}}{\frac{\Delta \theta_{j+1}}{k_{j+1}}+\frac{\Delta \theta_{j}}{k_{j}}} \\
& k_{j-1 / 2}=\frac{\Delta \theta_{j}+\Delta \theta_{j-1}}{\frac{\Delta \theta_{j}}{k_{j}}+\frac{\Delta \theta_{j-1}}{k_{j-1}}} \\
& k_{k+1 / 2}=\frac{\Delta z_{k+1}+\Delta z_{k}}{\frac{\Delta z_{k+1}}{k_{k+1}}+\frac{\Delta z_{k}}{k_{k}}} \\
& k_{k-1 / 2}=\frac{\Delta z_{k}+\Delta z_{k-1}}{\frac{\Delta z_{k}}{k_{k}}+\frac{\Delta z_{k-1}}{k_{k-1}}},
\end{aligned}
$$

which can then be used to assemble the face flux terms in Eq (35) with face areas:

$$
\begin{aligned}
A_{i+1 / 2} & =r_{i+1 / 2} \Delta \theta_{j} \Delta z_{k} \\
A_{i-1 / 2} & =r_{i-1 / 2} \Delta \theta_{j} \Delta z_{k} \\
A_{j+1 / 2} & =\Delta r_{i} \Delta z_{k} \\
A_{j-1 / 2} & =\Delta r_{i} \Delta z_{k} \\
A_{k+1 / 2} & =\frac{1}{2} \Delta \theta_{j}\left(r_{i+1 / 2}^{2}-r_{i-1 / 2}^{2}\right) \\
A_{k-1 / 2} & =\frac{1}{2} \Delta \theta_{j}\left(r_{i+1 / 2}^{2}-r_{i-1 / 2}^{2}\right) .
\end{aligned}
$$

On the $r$ and $z$ boundaries we want to be able to apply Dirichlet (fixed temperature) and Neumann (fixed heat flux) conditions. For Dirichlet conditions, the temperature on the boundary, $T_{b}$, is given resulting in the following face flux equations in $r$ and $z$ using a first-order reconstruction:

$$
\begin{aligned}
q_{i+1 / 2}^{b} & =-2 k_{i} \frac{T_{b}-T_{i}}{\Delta r_{i}} \\
q_{i-1 / 2}^{b} & =-2 k_{i} \frac{T_{i}-T_{b}}{\Delta r_{i}} \\
q_{k+1 / 2}^{b} & =-2 k_{k} \frac{T_{b}-T_{k}}{\Delta z_{k}} \\
q_{k-1 / 2}^{b} & =-2 k_{k} \frac{T_{k}-T_{b}}{\Delta z_{k}} .
\end{aligned}
$$

For Neumann conditions, the magnitude of the heat flux orthogonal the boundary, $q^{b}$, is prescribed such that:

$$
\mathbf{q} \cdot \mathbf{n}=-k q^{b},
$$

leading to the following face flux equations:

$$
\begin{aligned}
q_{i+1 / 2}^{b} & =-k_{i} q^{b} \\
q_{i-1 / 2}^{b} & =k_{i} q^{b} \\
q_{k+1 / 2}^{b} & =-k_{k} q^{b} \\
q_{k-1 / 2}^{b} & =k_{k} q^{b} .
\end{aligned}
$$


In general, a Dirichlet condition will be applied to the outer clad surface using the crud/clad interface temperature, $T_{c c}$, while a Neumann condition using the power calculated by the neutronics model, $q_{\phi}^{\prime \prime}$ is applied to the inner surface of the clad. The bottom and top of the cladding in the $z$ direction is assumed to be adiabatic with Neumann conditions set to zero.

Assembling the set of equations defined by Eq (28) for all cells using the derived flux terms and boundary conditions gives a linear system to be solved for the temperature:

$$
\mathbf{A T}=\mathbf{b},
$$

where $\mathbf{A}$ is the matrix of temperature coefficients from the flux equations, $\mathbf{b}$ are the forcing terms arising from boundary conditions, and $\mathbf{T}$ the vector of cell temperatures. Because of the cylindrical finite volume discretization this system is not symmetric due to the changing radius within a given cell. In this work GMRES was used to solve the linear system resulting from the discretization.

\subsubsection{Cladding Consumption}

Unlike the 1D model where the oxide and clad domains are clearly defined by the current oxide thickness, the 3D model requires constant material properties in every cell to satisfy the finite volume discretization. To do this, a material mixture is defined in every cell as a combination of oxide and clad material in a way that conserves total material mass as a function of the current oxide/metal interface. This is done under the assumption that the oxide consumes the cladding by growing inward from the cladding outer surface rather than both inward and outward. In addition, because a 1D corrosion model is used the oxide is treated as propagating through a $j k$ radial zone of the mesh in the $r$ direction. If the oxide/metal interface resides within a given cell with cardinal index $i j k$ the total volume is:

$$
V_{i j k}=\frac{1}{2} \Delta \theta_{j} \Delta k\left(r_{i+1 / 2}^{2}-r_{i-1 / 2}^{2}\right),
$$

and the volume of oxide in that cell is:

$$
V_{i j k}^{\text {oxide }}=\frac{1}{2} \Delta \theta_{j} \Delta z_{k}\left(r_{i+1 / 2}^{2}-\left(r_{o r}-S_{j k}\right)^{2}\right),
$$

where $r_{o r}$ is the outer radius of the cladding and $S_{j k}$ is the current oxide thickness in the $j k$ radial zone. It follows that the volume of clad material in a cell is then:

$$
V_{i j k}^{\text {clad }}=V_{i j k}-V_{i j k}^{\text {oxide }} .
$$

The density of the cell is:

$$
\rho_{i j k}=\frac{V_{i j k}^{\text {oxide }} \rho^{o x i d e}+V_{i j k}^{\text {clad }} \rho^{\text {clad }}}{V_{i j k}},
$$

which can then be used to compute a mass-weighted cell thermal conductivity:

$$
k_{i j k}=\left[\frac{\frac{1}{k^{\text {oxide }}} V_{i j k}^{\text {oxide }} \rho^{\text {oxide }}+\frac{1}{k^{\text {clad }}} V_{i j k}^{\text {clad }} \rho^{\text {clad }}}{V_{i j k} \rho_{i j k}}\right]^{-1} .
$$

As the cladding is consumed at every time step in Algorithm 1, the conductivities used in the thermal calculation must be updated with those calculated in Eq (49), requiring re-assembly of the linear operator and forcing vector in Eq (44) at every time step.

\subsubsection{Oxide-Metal Interface Temperature Evaluation}

Accurate reconstruction of the temperature at the metal/oxide interface is necessary for evaluation of the corrosion model. In the 1D model, this is evaluated analytically with knowledge of the interface location. In $3 \mathrm{D}$, due to the material smearing from cladding consumption as 
outlined in the previous section, a reconstruction is required at the interface to evaluate the temperature in a given cell using the current 3D temperature solution. Although the current interface location is still known, it will not be exact as it was computed using temperatures calculated from an inexact representation of the cladding materials.

Within a cell, we choose a local radial approximation to reconstruct the temperature. First, the heat flux on the $i-1 / 2$ face of the cell is reconstructed using the second-order representation and the conductivity computed from cladding consumption:

$$
q_{i-1 / 2}^{\prime \prime}=-2 k_{i j k} \frac{T_{i}-T_{i-1}}{\Delta r_{i}+\Delta r_{i-1}} .
$$

Note that if the $i-1 / 2$ face of the cell is on the inner clad radius boundary then the boundary condition is used for $q_{i-1 / 2}^{\prime \prime}$. Next, a $1 \mathrm{D}$ conduction problem is created assuming that the cell temperature, $T_{i j k}$, is located at the center of the cell. First the energy entering the cell from the $i-1 / 2$ face is computed:

$$
Q_{i-1 / 2}=q_{i-1 / 2}^{\prime \prime} A_{i-1 / 2}
$$

which gives coefficients:

$$
\begin{aligned}
c_{1_{i j k}} & =-\frac{Q_{i-1 / 2}}{k_{i j k} \Delta \theta_{j} \Delta z_{k}} \\
c_{2_{i j k}} & =T_{i j k}-c_{1_{i j k}} \ln \left(r_{i}\right),
\end{aligned}
$$

that can be used to evaluate the temperature at a radial location, $r$, within a cell:

$$
T_{i j k}(r)=c_{1_{i j k}} \ln (r)+c_{2_{i j k}} .
$$

If the oxide/metal interface is in the cell, the oxide/metal interface temperature for the current radial zone with oxide thickness $S_{j k}$ is then:

$$
T_{o m_{j k}}=c_{1_{i j k}} \ln \left(r_{o r}-S_{j k}\right)+c_{2_{i j k}} .
$$

Although we used a radial reconstruction here, it would be entirely possible to build 3-dimensional interpolant from a gradient reconstruction method which incorporates information from cells in the $\theta$ and $z$ directions.

\subsubsection{Surface Heat Flux Reconstruction}

To couple back to the fluid calculation, we need to reconstruct the return surface heat flux, $q_{\text {surf }}^{\prime \prime}$, from the computed temperatures. We do this using a first-order flux reconstruction along with the prescribed Dirichlet condition on the outer radius of the clad surface. For a cell $i j k$ on the boundary this amounts to computing the heat flux on the $i+1 / 2$ face using the boundary condition as the $T_{i+1 / 2}$ temperature:

$$
q_{s u r f}^{\prime \prime}=-2 k_{i j k} \frac{T_{c c_{i j k}}-T_{i j k}}{\Delta r_{i}}
$$

where $T_{c c_{i j k}}$ is the clad/crud interface temperature on the $i j k$ cell surface.

\subsection{Computational Tool}

The 1D and 3D thermal models along with coupling infrastructure for corrosion and crud models were added to Cicada to achieve the coupled scheme outlined in Algorithm 1. There are 4 primary abstractions: boundary conditions for coupling the models and the crud model, corrosion model, and clad model. 


\subsubsection{Boundary Condition Interface and STAR-CCM+ Coupling}

Boundary conditions are the core Cicada concept for coupling models at shared interfaces. In particular, they were designed to for use with DTK to couple shared boundary data on different grids with different parallel partitioning. Cicada boundary conditions expose the spatial coordinates at which to perform the coupling, in this case the centroids of the mesh faces on the shared interface, access to the data on those faces, and the areas of the faces. Along with a host of other functions for accessing and manipulating the boundary condition, the core coupling interface is defined in the following.

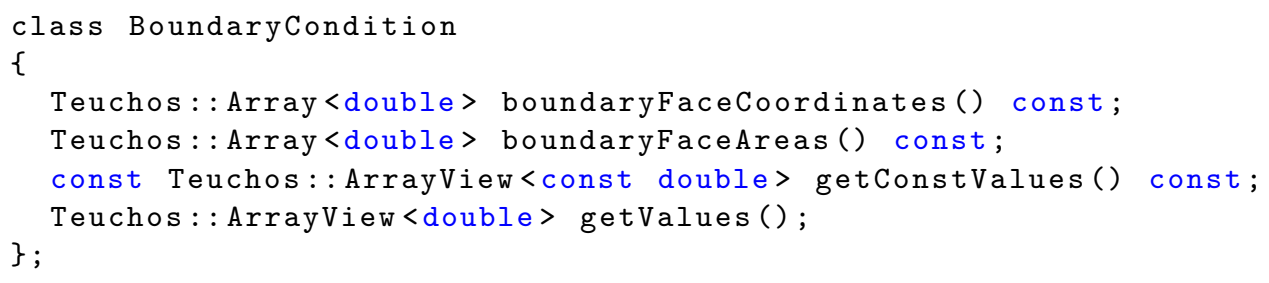

This interface presumes that face values are constant, which is the case for STAR-CCM + , the crud and corrosion models, and both 1D and 3D thermal model discretizations. This information can be used to couple boundaries using DTK's mesh-free surface transfer capability. As shown in [6], these algorithms are particularly effective for boundaries with curvature such as the fuel rod surface. In addition, the face area values can be used to enforce global conservation of transferred quantities on the coupling surface.

The objective of this work is to analyze the behavior of the cladding models in the context of a single conjugate heat transfer iteration. Therefore, STAR-CCM+ coupling can be done offline with files for the purpose of completing this analysis. Using the aforementioned HDF5 capability developed for reading and writing STAR-CCM+ data for further analysis, the rod wetted surface conditions can be reconstructed within a given boundary condition using DTK using data directly from the HDF5 file. This includes surface temperature and turbulent kinetic energy. It should be noted that this strategy can be easily adapted to couple the clad models to STAR-CCM+ in-memory for further algorithmic assessment of full conjugate heat transfer as the HDF5 data is structured in a nearly identical fashion to that provided by the STAR-CCM+ coupling interface.

\subsubsection{Crud Model Interface}

MAMBA 1D was wrapped in a new $\mathrm{C}++$ interface for this work to facilitate integration into the coupling scheme presented in Algorithm 1. Instead of growing crud on the surface of the CFD model mesh, the new scheme grows crud on the structured cylindrical grid used for the thermal calculations to facilitate coupling within the clad model. In addition, it provides a means of exploring the spatial fidelity needed in the crud model to capture local effects from the CFD-defined boundary conditions reconstructed on the outer surface of the crud using the DTK interpolation described above. The crud model interface abstracts MAMBA 1D as a general transient model with accessors to model responses and boundary conditions: 


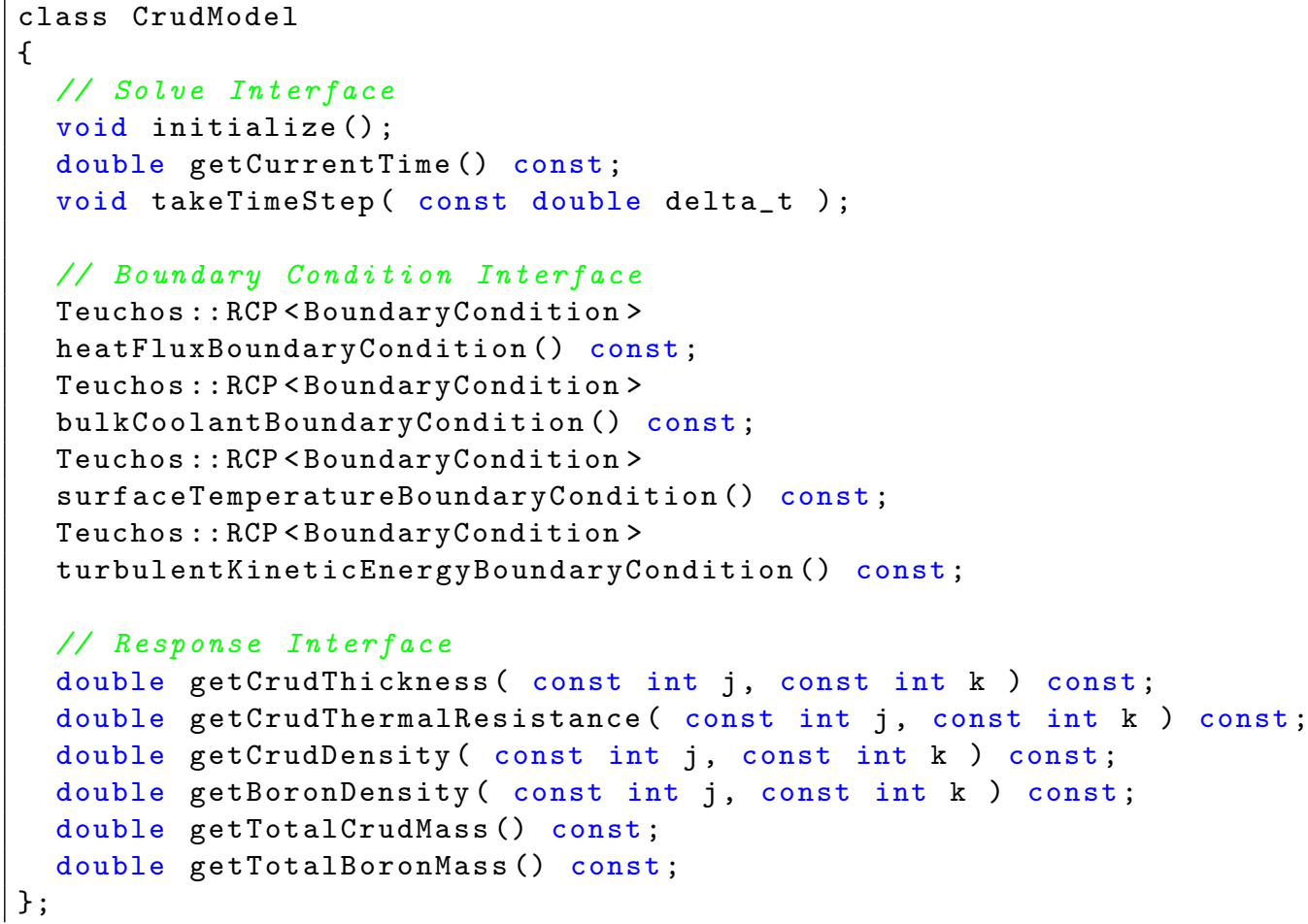

This interface will be easily adaptable to MAMBA 3D or similar 3D crud variants once they have been refactored to execute as a library and integrated into VERA. For 3D crud this interface would remain nearly identical. Instead of using $j k$ indices in the response interface, which can be used here because the crud and thermal grids are identical, coupling to the corrosion and thermal models can be achieved with an additional boundary condition for the crud thermal resistance. This would allow for general coupling to the thermal model to allow for any mesh to be used for the 3D crud model.

\subsubsection{Corrosion Model Interface}

Although the EPRI model described above was used for this work, the model was abstracted in a way that any 1D, continuum-scale corrosion model could be used within the coupling sequence. The following interface was developed to evaluate the oxide thickness over given time step given a current oxide thickness and the temperature at the oxide/metal interface.

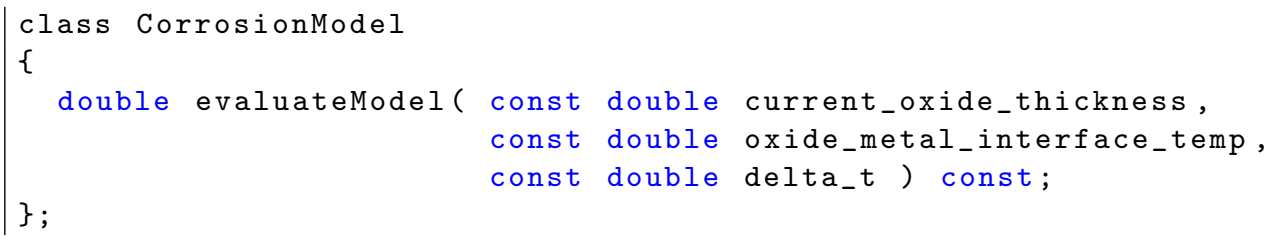

The interface can be updated in the future to include a variety of model additional model parameters and coupling inputs. This will allow for various models to be interchanged for assessment within the coupling algorithm.

\subsubsection{Clad Model Interface}

In Cicada the corrosion and thermal models are combined to create a single model for the cladding material which is then coupled to the crud model. This permits interchanging the 1D and 3D models and their different means of including the effects of oxidation of the cladding. The following interface is defined. 


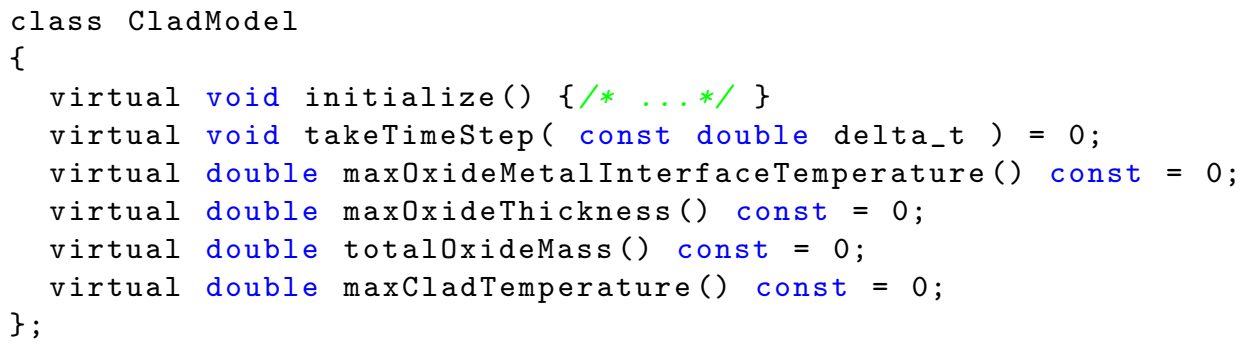

Both 1D and 3D subclasses are implemented that override the virtual functions.

\subsection{CFD Model for Analysis}

A single pin model provided by the University of Michigan was used for the algorithmic assessment in this work with the properties given in Table 4 . The model contains three spacer grids

\section{Table 4 . Single pin at $160 \%$ nominal power specification.}

\begin{tabular}{|l|c|}
\hline Rod height & $365.76[\mathrm{~cm}]$ \\
\hline Rod outer radius & $0.475[\mathrm{~cm}]$ \\
\hline Pin pitch & $1.6[\mathrm{~cm}]$ \\
\hline Inlet mass flow rate & $0.3[\mathrm{~kg} / \mathrm{s}]$ \\
\hline Inlet temperature & $565.85[\mathrm{~K}]$ \\
\hline Total integrated power (flat profile) & $6.69304 \mathrm{E}+04[W]$ \\
\hline
\end{tabular}

at the top of the pin and includes the Rosenhow boiling model. The model was run for the fluid only with varying levels of total pin powers - heat transfer in the fuel, gap, and clad was not modeled. The power from fission was evenly provided both axially and azimuthally on the outer surface of the cladding. Heat flux profiles with axial and azimuthal dependence will be analyzed in future work.

\subsection{Time Integration}

The model defined in Algorithm 1 is expected to be expensive to evaluate. For example, a cylindrical clad mesh at CFD length scales could contain millions of cells even with such a thin structure in the radial direction. Therefore, it is critical to seek ways to reduce the computational cost of the model while retaining the fidelity of the solution. In terms of time integration, the cost is also high with crud time steps restricted to $O(1 e 4)$ seconds when growth is to be resolved at CFD length scales. Simulating 500-1000 days of crud growth and oxidation therefore requires thousands to more than ten thousand time steps, each of which contain an evaluation of the crud, corrosion, and thermal models.

In the clad model, the corrosion and thermal models in 1D are effectively combined while the 3D model requires separate evaluation of the corrosion model and thermal model with homogenization and reconstruction used to couple them together. To reduce computational cost, we will assess sub-cycling the crud model such that multiple crud time steps occur for every corrosion/thermal time step. Shown in Algorithm 2, sub-cycling the crud model amounts to adding an additional for loop around the model evaluation. 


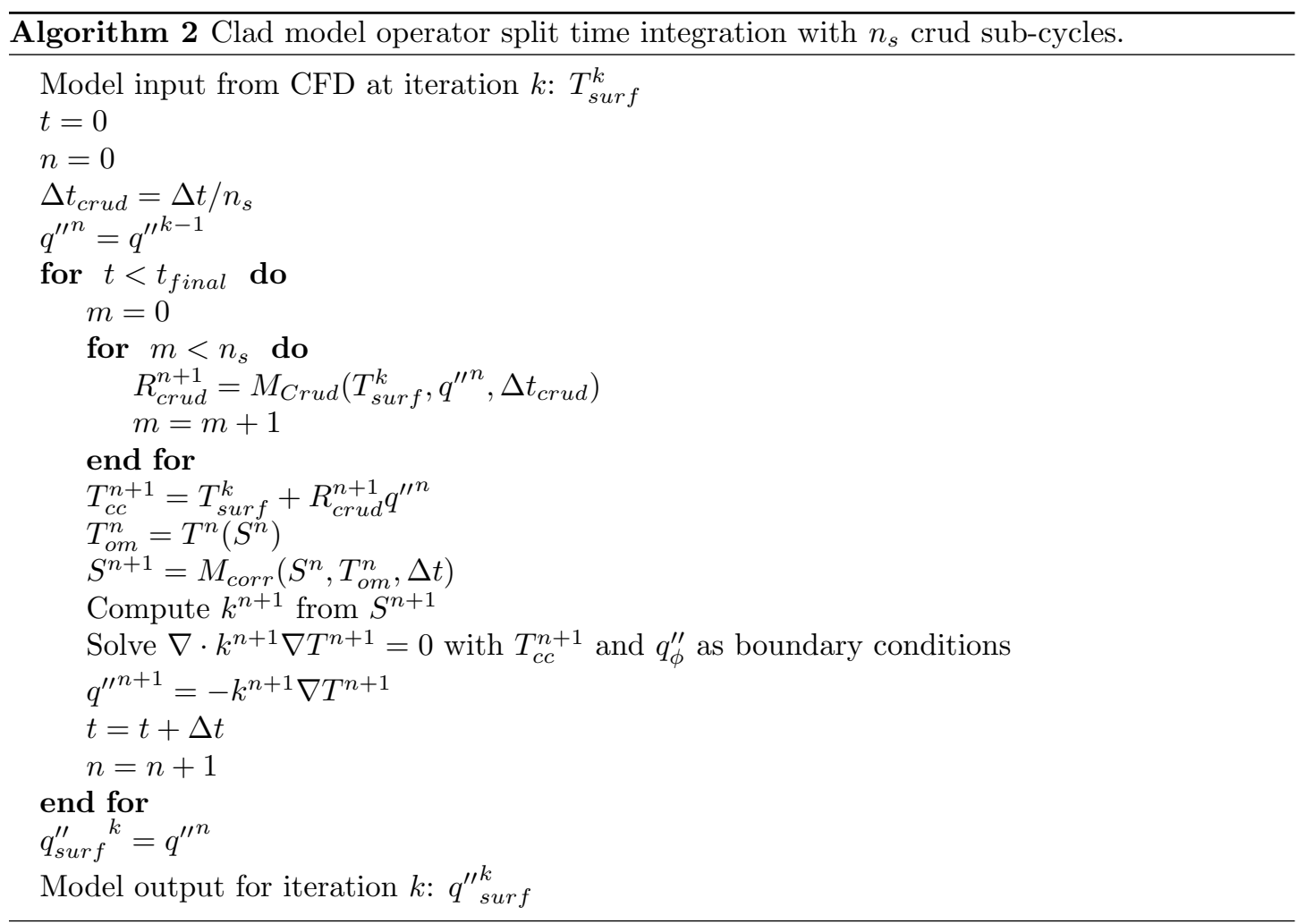

Using STAR-CCM+ boundary conditions from a single pin solve at $180 \%$ nominal power and corresponding $q_{\phi}^{\prime \prime}$ boundary condition for the thermal solve the amount of crud sub-cycling was varied to assess the tradeoff between accuracy and time to solution using the 1D clad model. Shown in Table 5, identical crud results and good oxidation and thermal results can be obtained with 40-50 crud sub-cycles in Algorithm 2 while reducing computational time over Algorithm 1 (i.e. 1 sub-cycle) by a factor of 3 . Above 40 sub-cycles the computational time is not substantially reduced with the 1D model, indicating that the majority of the remaining run time is spent evaluating the crud model and therefore we will use 40 sub-cycles for the remainder of this work.

Table 5. 1D model crud sub-cycling results. The crud model time step was fixed at 1.0e4 seconds while the clad model time step increases with sub-cycle count.

\begin{tabular}{|c|c|c|c|c|c|c|c|}
\hline Crud Sub-cycles & 1 & 5 & 10 & 20 & 40 & 50 & 100 \\
\hline Clad Time Step (days) & 0.12 & 0.58 & 1.16 & 2.31 & 4.63 & 5.79 & 11.57 \\
\hline Max Crud Thickness (m) & $1.501 \mathrm{E}-04$ & $1.501 \mathrm{E}-04$ & $1.501 \mathrm{E}-04$ & $1.501 \mathrm{E}-04$ & $1.501 \mathrm{E}-04$ & $1.501 \mathrm{E}-04$ & $1.501 \mathrm{E}-04$ \\
\hline Total Boron Mass (kg) & $2.483 \mathrm{E}-05$ & $2.483 \mathrm{E}-05$ & $2.483 \mathrm{E}-05$ & $2.483 \mathrm{E}-05$ & $2.483 \mathrm{E}-05$ & $2.483 \mathrm{E}-05$ & $2.483 \mathrm{E}-05$ \\
\hline Max Metal/Oxide Interface Temp (K) & 643.13 & 643.152 & 643.173 & 643.214 & 643.115 & 643.318 & 643.797 \\
\hline Max Oxide Thickness (microns) & 20.7148 & 20.7445 & 20.7724 & 20.8282 & 20.6946 & 20.9698 & 21.6181 \\
\hline
\end{tabular}

More sub-cycles could arguably be used based on these results, however, we will cautiously remain on the side of better time accuracy in this work to ensure accurate tracking of the oxide/metal interface in the 3D clad model. It should also be noted that speed-ups obtained from sub-cycling using the 3D model are even more substantial due to the additional cost of the 3D thermal solve. Not sub-cycling the crud with the 3D model and therefore performing thousands of 3D solves was found to effectively make the problem intractable for fine grids. 


\subsection{Preconditioning}

Preconditioning the linear system in Eq (44) resulting from the 3D discretization of the thermal problem is critical to obtaining a reasonable time to solution by reducing the number of GMRES iterations required for convergence. In addition, because the cladding material properties change with time as the result of oxidation, the matrix coefficients in the linear system also change, resulting in the need to recompute the preconditioner before every thermal solve. This leads to the time needed to construct the preconditioner also being an important factor.

To alleviate some of the setup cost of the preconditioner, we investigate reusing the preconditioner for a specified number of thermal solves before we rebuild it. This is valid under the assumption that the properties operator do not change dramatically from time step to time step corresponding with relatively slow propagation of the oxide through the cladding. At some point, iterative performance of the preconditioner will decrease enough that it is beneficial to rebuild it (this is often referred to as the preconditioner becoming "stale").

Table 6 gives the timing results for varying levels of preconditioner use using an ILUT preconditioner with a fill level of 1 the the GMRES convergence criteria set to 1.0e- 4 . In these calculations the STAR-CCM+ boundary conditions from a single pin solve at $180 \%$ nominal power were again used with the corresponding $q_{\phi}^{\prime \prime}$ boundary condition for the thermal solve. These results indicate that some reuse of the preconditioner is beneficial to reducing runtime. Furthermore, if it was found that the number of crud sub-cycles had to be reduced due to time integration constraints, reductions in timing by preconditioner reuse would improve further as the total number of thermal model evaluations would increase.

Table 6. 3D model preconditioner reuse results with ILUT(1).

\begin{tabular}{llll}
\hline Solves per Preconditioner Rebuild & 1 & 5 & 10 \\
\hline Serial Run Time (s) & 954 & 934 & 1049 \\
\hline
\end{tabular}

\subsection{D Mesh Refinement}

A mesh refinement study was performed with the 1D clad model to assess the spatial resolution needed in $\theta$ and $z$ to capture the local effects from the STAR-CCM+ boundary conditions. A baseline fine grid of 128 cells in the azimuthal direction and 1000 cells in the radial direction was assumed. First, using the $180 \%$ power case the $z$ grid was fixed at 1000 cells and the number of cells in the $\theta$ direction was varied from 4 to 128 as shown in Table 7 . Using 16 cells is sufficient for this case to capture the total oxide mass within a few digits, 32 cells needed for the total boron and crud mass, while 64 cells performs better for the maximum local crud and oxide thicknesses.

Table 7. 1D model theta mesh refinement. Z cells fixed at 1000 .

\begin{tabular}{lllllll}
\hline Theta Cells & 4 & 8 & 16 & 32 & 64 & 128 \\
\hline Max Crud Thickness (m) & $1.496 \mathrm{E}-04$ & $1.153 \mathrm{E}-04$ & $1.406 \mathrm{E}-04$ & $1.488 \mathrm{E}-04$ & $1.499 \mathrm{E}-04$ & $1.500 \mathrm{E}-04$ \\
Total Crud Mass (kg) & $3.249 \mathrm{E}-03$ & $3.540 \mathrm{E}-03$ & $3.583 \mathrm{E}-03$ & $3.578 \mathrm{E}-03$ & $3.576 \mathrm{E}-03$ & $3.577 \mathrm{E}-03$ \\
Total Boron Mass (kg) & $2.767 \mathrm{E}-05$ & $2.459 \mathrm{E}-05$ & $2.525 \mathrm{E}-05$ & $2.475 \mathrm{E}-05$ & $2.476 \mathrm{E}-05$ & $2.477 \mathrm{E}-05$ \\
Max Metal/Oxide Interface Temp $(\mathrm{K})$ & 643.075 & 639.455 & 642.156 & 642.976 & 643.098 & 643.114 \\
Max Oxide Thickness (microns) & 20.672 & 18.5987 & 20.2061 & 20.6208 & 20.6836 & 20.6918 \\
Total Oxide Mass (kg) & $7.607 \mathrm{E}-03$ & $7.933 \mathrm{E}-03$ & $7.950 \mathrm{E}-03$ & $7.948 \mathrm{E}-03$ & $7.947 \mathrm{E}-03$ & $7.947 \mathrm{E}-03$ \\
Max Clad Temperature (K) & 699.567 & 696.053 & 698.671 & 699.469 & 699.589 & 699.604 \\
Serial Run Time (s) & 3.2 & 4.9 & 9.3 & 18 & 36 & 72 \\
\hline
\end{tabular}

Next, a refinement is $z$ was performed with the number of $\theta$ cells fixed at 128 as shown in Table 8. We see that the 250 cells in $z$ is sufficient to capture maximum crud and oxide thicknesses as well as total crud and boron mass while 500 cells is needed for total crud thickness. 
Table 8. 1D model $\mathrm{Z}$ mesh refinement. Theta cells fixed at 128 .

\begin{tabular}{lllll}
\hline Z Cells & 100 & 250 & 500 & 1000 \\
\hline Max Crud Thickness (m) & $1.476 \mathrm{E}-04$ & $1.500 \mathrm{E}-04$ & $1.501 \mathrm{E}-04$ & $1.500 \mathrm{E}-04$ \\
Total Crud Mass (kg) & $3.604 \mathrm{E}-03$ & $3.565 \mathrm{E}-03$ & $3.576 \mathrm{E}-03$ & $3.577 \mathrm{E}-03$ \\
Total Boron Mass (kg) & $2.472 \mathrm{E}-05$ & $2.474 \mathrm{E}-05$ & $2.475 \mathrm{E}-05$ & $2.477 \mathrm{E}-05$ \\
Max Metal/Oxide Interface Temp (K) & 642.864 & 643.116 & 643.116 & 643.114 \\
Max Oxide Thickness (microns) & 20.564 & 20.6927 & 20.6949 & 20.6918 \\
Total Oxide Mass (kg) & $7.954 \mathrm{E}-03$ & $7.949 \mathrm{E}-03$ & $7.947 \mathrm{E}-03$ & $7.947 \mathrm{E}-03$ \\
Max Clad Temperature (K) & 699.36 & 699.606 & 699.606 & 699.604 \\
Serial Run Time (s) & 7.6 & 18 & 36 & 72 \\
\hline
\end{tabular}

Finally, we perform a coarse calculation with $32 \theta$ cells and $250 z$ cells and compare to fine result in Table 9 . The results show good agreement while substantially reducing run time.

Table 9. 1D model mesh refinement. Coarse (Theta $=32, \mathrm{Z}=250$ ) vs. fine (Theta $=128, \mathrm{Z}=1000$ ).

\begin{tabular}{lll}
\hline & Coarse & Fine \\
\hline Max Crud Thickness (m) & $1.486 \mathrm{E}-04$ & $1.500 \mathrm{E}-04$ \\
Total Crud Mass (kg) & $3.565 \mathrm{E}-03$ & $3.577 \mathrm{E}-03$ \\
Total Boron Mass (kg) & $2.467 \mathrm{E}-05$ & $2.477 \mathrm{E}-05$ \\
Max Metal/Oxide Interface Temp (K) & 642.96 & 643.114 \\
Max Oxide Thickness (microns) & 20.6131 & 20.6918 \\
Total Oxide Mass (kg) & $7.949 \mathrm{E}-03$ & $7.947 \mathrm{E}-03$ \\
Max Clad Temperature (K) & 699.455 & 699.604 \\
Serial Run Time (s) & 3.35 & 72 \\
\hline
\end{tabular}

\subsection{D Mesh Refinement}

We next repeat the mesh refinement analysis for the 3D model. Table 10 gives the results for uniform mesh refinement in the radial direction with a fixed number of 16 cells in the $\theta$ direction and 250 cells in the $z$ direction for the $180 \%$ power case. We note that at coarse radial discretizations, there is a large error compared to the fine discretization due to the smearing of oxide materials and reconstruction error in larger radial zones. As the radial mesh is refined, the oxide/metal interface is more accurately tracked. From these results we see that at least a half micron mesh resolution is needed to resolve oxidation.

Table 10. 3D model uniform radial mesh refinement.

\begin{tabular}{|c|c|c|c|c|c|c|c|c|}
\hline R Cells & 10 & 25 & 50 & 100 & 200 & 500 & 1000 & 2000 \\
\hline Max Crud Thickness (m) & $1.403 \mathrm{E}-04$ & $1.403 \mathrm{E}-04$ & $1.403 \mathrm{E}-04$ & $1.403 \mathrm{E}-04$ & $1.403 \mathrm{E}-04$ & $1.403 \mathrm{E}-04$ & $1.403 \mathrm{E}-04$ & $1.403 \mathrm{E}-04$ \\
\hline Total Crud Mass (kg) & $3.567 \mathrm{E}-03$ & $3.568 \mathrm{E}-03$ & $3.567 \mathrm{E}-03$ & $3.567 \mathrm{E}-03$ & $3.567 \mathrm{E}-03$ & $3.568 \mathrm{E}-03$ & $3.567 \mathrm{E}-03$ & $3.568 \mathrm{E}-03$ \\
\hline Total Boron Mass (kg) & $2.527 \mathrm{E}-05$ & $2.530 \mathrm{E}-05$ & $2.527 \mathrm{E}-05$ & $2.526 \mathrm{E}-05$ & $2.525 \mathrm{E}-05$ & $2.526 \mathrm{E}-05$ & $2.526 \mathrm{E}-05$ & $2.526 \mathrm{E}-05$ \\
\hline Max Metal/Oxide Interface Temp (K) & 629.647 & 632.46 & 638.425 & 639.819 & 640.527 & 641.209 & 641.35 & 641.441 \\
\hline Max Oxide Thickness (microns) & 16.2352 & 16.7607 & 17.8498 & 18.8683 & 19.3776 & 19.9017 & 19.9946 & 20.0417 \\
\hline Total Oxide Mass $(\mathrm{kg})$ & $6.782 \mathrm{E}-03$ & $6.892 \mathrm{E}-03$ & $7.092 \mathrm{E}-03$ & $7.473 \mathrm{E}-03$ & $7.670 \mathrm{E}-03$ & $7.855 \mathrm{E}-03$ & $7.897 \mathrm{E}-03$ & $7.920 \mathrm{E}-03$ \\
\hline Max Clad Temperature (K) & 694.643 & 695.062 & 696.15 & 696.889 & 697.264 & 697.637 & 697.708 & 697.739 \\
\hline Serial Run Time (s) & 7 & 14 & 24 & 44 & 95 & 275.7 & 623 & 1474 \\
\hline
\end{tabular}

Next we consider refining the grid in the radial direction with a fixed fine grid over the region 
of oxidation, effectively equivalent to a boundary layer mesh, to more accurately capture the oxide/metal interface while using a smaller number of cells in the radial direction. Assuming an oxidation zone of 25 microns for the $180 \%$ power case a fixed mesh of 50 cells of 0.5 micron radial spacing was used for the oxide while the number of radial cells in the remainder of the cladding material was varied. Table 11 gives the results of these calculations. Once the oxide region has been resolved with a fine grid, only a handful of cells are needed in the un-oxidized cladding region to achieve an accurate solution. In fact, the difference between 5 and 50 cells was negligible. Using only 5 cells to resolve the clad region with 50 cells in the oxide region gave effectively the same results as the finest radial discretization given in Table 10 with more than a factor of 20 reduction in run time.

Table 11. 3D model radial mesh refinement with oxide layer. Oxide mesh is 25 microns thick with 0.5 micron mesh spacing.

\begin{tabular}{lllll}
\hline R Clad Cells & 5 & 10 & 20 & 50 \\
\hline Max Crud Thickness (m) & $1.403 \mathrm{E}-04$ & $1.403 \mathrm{E}-04$ & $1.403 \mathrm{E}-04$ & $1.403 \mathrm{E}-04$ \\
Total Crud Mass (kg) & $3.568 \mathrm{E}-03$ & $3.567 \mathrm{E}-03$ & $3.568 \mathrm{E}-03$ & $3.567 \mathrm{E}-03$ \\
Total Boron Mass (kg) & $2.525 \mathrm{E}-05$ & $2.526 \mathrm{E}-05$ & $2.525 \mathrm{E}-05$ & $2.526 \mathrm{E}-05$ \\
Max Metal/Oxide Interface Temp (K) & 641.442 & 641.441 & 641.441 & 641.441 \\
Max Oxide Thickness (microns) & 20.0419 & 20.0417 & 20.0417 & 20.0417 \\
Total Oxide Mass (kg) & $7.920 \mathrm{E}-03$ & $7.920 \mathrm{E}-03$ & $7.920 \mathrm{E}-03$ & $7.920 \mathrm{E}-03$ \\
Max Clad Temperature (K) & 697.682 & 697.725 & 697.736 & 697.739 \\
Serial Run Time (s) & 38 & 42 & 49 & 69 \\
\hline
\end{tabular}

Using the fine radial mesh in the oxidized region and a coarse radial mesh in the remaining cladding, refinement in $\theta$ and $z$ was performed with the results given in Table 12 and Table 13 respectively. These results indicate, similar to the $1 \mathrm{D}$ results, that 32 cells in the $\theta$ direction and 250 cells in the $z$ direction are sufficient to capture the effects of the CFD boundary condition on the clad model.

Table 12. 3D model theta mesh refinement with oxide layer. Oxide mesh is 25 microns thick with 0.5 micron mesh spacing. $5 \mathrm{R}$ cells in remaining cladding and $250 \mathrm{Z}$ cells.

\begin{tabular}{llllll}
\hline Theta Cells & 8 & 16 & 32 & 64 & 128 \\
\hline Max Crud Thickness (m) & $1.154 \mathrm{E}-04$ & $1.403 \mathrm{E}-04$ & $1.485 \mathrm{E}-04$ & $1.499 \mathrm{E}-04$ & $1.501 \mathrm{E}-04$ \\
Total Crud Mass (kg) & $3.527 \mathrm{E}-03$ & $3.568 \mathrm{E}-03$ & $3.563 \mathrm{E}-03$ & $3.563 \mathrm{E}-03$ & $3.562 \mathrm{E}-03$ \\
Total Boron Mass (kg) & $2.461 \mathrm{E}-05$ & $2.525 \mathrm{E}-05$ & $2.467 \mathrm{E}-05$ & $2.475 \mathrm{E}-05$ & $2.474 \mathrm{E}-05$ \\
Max Metal/Oxide Interface Temp (K) & 639.081 & 641.442 & 642.044 & 642.135 & 642.165 \\
Max Oxide Thickness (microns) & 18.5392 & 20.0419 & 20.3795 & 20.4449 & 20.4599 \\
Total Oxide Mass (kg) & $7.908 \mathrm{E}-03$ & $7.920 \mathrm{E}-03$ & $7.918 \mathrm{E}-03$ & $7.918 \mathrm{E}-03$ & $7.918 \mathrm{E}-03$ \\
Max Clad Temperature (K) & 695.786 & 697.682 & 697.922 & 697.983 & 698.013 \\
Serial Run Time (s) & 18 & 38 & 80 & 171 & 377 \\
\hline
\end{tabular}


Table 13. 3D model $\mathrm{Z}$ mesh refinement with oxide layer. Oxide mesh is 25 microns thick with 0.5 micron mesh spacing. $5 \mathrm{R}$ cells in remaining cladding and $32 \mathrm{Z}$ cells.

\begin{tabular}{lllll}
\hline Z Cells & 100 & 250 & 500 & 1000 \\
\hline Max Crud Thickness (m) & $1.464 \mathrm{E}-04$ & $1.485 \mathrm{E}-04$ & $1.487 \mathrm{E}-04$ & $1.487 \mathrm{E}-04$ \\
Total Crud Mass (kg) & $3.603 \mathrm{E}-03$ & $3.563 \mathrm{E}-03$ & $3.574 \mathrm{E}-03$ & $3.577 \mathrm{E}-03$ \\
Total Boron Mass (kg) & $2.462 \mathrm{E}-05$ & $2.467 \mathrm{E}-05$ & $2.472 \mathrm{E}-05$ & $2.477 \mathrm{E}-05$ \\
Max Metal/Oxide Interface Temp (K) & 641.878 & 642.044 & 642.052 & 642.052 \\
Max Oxide Thickness (microns) & 20.2724 & 20.3795 & 20.3863 & 20.3857 \\
Total Oxide Mass (kg) & $7.924 \mathrm{E}-03$ & $7.918 \mathrm{E}-03$ & $7.916 \mathrm{E}-03$ & $7.916 \mathrm{E}-03$ \\
Max Clad Temperature (K) & 697.745 & 697.922 & 697.928 & 697.928 \\
Serial Run Time (s) & 31 & 80 & 185 & 491 \\
\hline
\end{tabular}

\subsection{Model Comparison}

The 1D and 3D models were directly compared on grids with 32 and 250 cells in the $\theta$ and $z$ directions respectively using the crud and corrosion model parameters given in Table 2 and Table 3 with a value of $9.0 \mathrm{e} 14$ for the neutron fast flux. These calculations were performed at $100 \%, 120 \%, 140 \%, 160 \%$, and $180 \%$ nominal pin power with a total nominal pin power of 6.69304e4 Watts. A simple flat power profile was used in these calculations, both to generate the CFD results as well as for the clad model boundary condition, $q_{\phi}^{\prime \prime}$. Tables $14,15,16,17$, and 18 give the results for each power level. These results show that for relatively thin oxide layers (12-20 microns in these cases), both the 3D and 1D models produce effectively the same results in total integrated values and global extrema for the crud, corrosion, and thermal models. As expected, the 3D model allows for thermal diffusion around the local resistance introduced by crud growth and oxidation therefore resulting in lower clad temperatures, oxide/metal interface temperatures, and reduced oxide growth. However, these effects on the order of $1 \%$ for each of the power levels.

The additional heat flow in the radial and azimuthal directions is more obvious in the results for coupling flux $\left(q_{\text {surf }}^{\prime \prime}\right)$ given in the tables. Due to the flat power profile the 1D result has no variation in the output surface flux - every radial zone on the interior surface of the clad received the same amount of power and therefore emitted the same amount as well. In the 3D calculation, the heat flow around local crud growth and oxidation creates local hot spots of flux up to $10 \%$ greater than the $1 \mathrm{D}$ case and local cool spots up to $20 \%$ smaller. This difference warrants investigating the use of both $1 \mathrm{D}$ and $3 \mathrm{D}$ models in a fully coupled conjugate heat transfer simulation with STAR-CCM+ to assess the feedback effects of these differences. If local variations in $q_{\text {surf }}^{\prime \prime}$ have a strong effect on surface temperatures computed by CFD $\left(T_{\text {surf }}\right)$ then use of a full 3D heat transfer model will be needed as crud and corrosion models are clearly very sensitive to local temperatures. For example, a $20 \%$ change in local heat flux between the $100 \%$ and $120 \%$ power cases given in Tables 17 and 18 result in an order of magnitude increase in boron deposition due to local temperatures above saturation and nearly a $20 \%$ of total oxide mass on the rod. If it is found that these local variations do not have a strong effect on surface temperatures, then a 1D thermal model would be deemed sufficient for driving the crud and corrosion models. 
Table 14. 3D vs. $1 \mathrm{D}$ model comparison at $100 \%$ power for 500 days.

\begin{tabular}{llll}
\hline & 1D & 3D & $\%$ Difference \\
\hline Max Surface Temperature (K) & 618.856 & 618.856 & \\
Min Surface Temperature (K) & 578.33 & 578.33 & \\
Max Crud Thickness (m) & $1.5778 \mathrm{E}-05$ & $1.5590 \mathrm{E}-05$ & $1.209 \%$ \\
Total Crud Mass (kg) & $2.5365 \mathrm{E}-04$ & $2.5364 \mathrm{E}-04$ & $0.005 \%$ \\
Total Boron Mass (kg) & $1.8673 \mathrm{E}-07$ & $1.8667 \mathrm{E}-07$ & $0.036 \%$ \\
Max Crud/Clad Interface Temp (K) & 619.649 & 619.642 & $0.001 \%$ \\
Max Coupling Flux (W/m^2) & $6.123 \mathrm{E}+05$ & $6.846 \mathrm{E}+05$ & $-10.552 \%$ \\
Min Coupling Flux (W/m^2) & $6.123 \mathrm{E}+05$ & $4.772 \mathrm{E}+05$ & $28.315 \%$ \\
Max Metal/Oxide Interface Temp (K) & 624.621 & 624.329 & $0.047 \%$ \\
Max Oxide Thickness (microns) & 12.1807 & 12.1346 & $0.380 \%$ \\
Total Oxide Mass (kg) & $2.953 \mathrm{E}-03$ & $2.946 \mathrm{E}-03$ & $0.217 \%$ \\
Max Clad Temperature (K) & 656.248 & 655.461 & $0.120 \%$ \\
Serial Run Time (s) & 5 & 69 & \\
\hline
\end{tabular}

Table 15. 3D vs. 1D model comparison at $120 \%$ power for 500 days.

\begin{tabular}{llll}
\hline & $1 \mathrm{D}$ & $3 \mathrm{D}$ & $\%$ Difference \\
\hline Max Surface Temperature (K) & 619.951 & 619.951 & \\
Min Surface Temperature (K) & 580.821 & 580.821 & \\
Max Crud Thickness (m) & $3.0061 \mathrm{E}-05$ & $3.0045 \mathrm{E}-05$ & $0.053 \%$ \\
Total Crud Mass (kg) & $5.3801 \mathrm{E}-04$ & $5.3781 \mathrm{E}-04$ & $0.037 \%$ \\
Total Boron Mass (kg) & $3.1147 \mathrm{E}-07$ & $3.1127 \mathrm{E}-07$ & $0.062 \%$ \\
Max Crud/Clad Interface Temp (K) & 620.393 & 620.39 & $0.000 \%$ \\
Max Coupling Flux (W/m^2) & $7.348 \mathrm{E}+05$ & $8.218 \mathrm{E}+05$ & $-10.585 \%$ \\
Min Coupling Flux (W/m^2) & $7.348 \mathrm{E}+05$ & $5.746 \mathrm{E}+05$ & $27.886 \%$ \\
Max Metal/Oxide Interface Temp (K) & 626.811 & 626.628 & $0.029 \%$ \\
Max Oxide Thickness (microns) & 13.0831 & 13.0548 & $0.217 \%$ \\
Total Oxide Mass (kg) & $4.246 \mathrm{E}-03$ & $4.234 \mathrm{E}-03$ & $0.290 \%$ \\
Max Clad Temperature (K) & 664.732 & 664.533 & $0.030 \%$ \\
Serial Run Time (s) & 5 & 72 & \\
\hline
\end{tabular}


Table 16. 3D vs. 1D model comparison at $140 \%$ power for 500 days.

\begin{tabular}{llll}
\hline & $1 \mathrm{D}$ & $3 \mathrm{D}$ & $\%$ Difference \\
\hline Max Surface Temperature (K) & 621.13 & 621.13 & \\
Min Surface Temperature (K) & 583.311 & 583.311 & \\
Max Crud Thickness (m) & $4.8140 \mathrm{E}-05$ & $4.7710 \mathrm{E}-05$ & $0.901 \%$ \\
Total Crud Mass (kg) & $1.1523 \mathrm{E}-03$ & $1.1515 \mathrm{E}-03$ & $0.074 \%$ \\
Total Boron Mass (kg) & $6.2496 \mathrm{E}-07$ & $6.2442 \mathrm{E}-07$ & $0.087 \%$ \\
Max Crud/Clad Interface Temp (K) & 621.311 & 621.271 & $0.006 \%$ \\
Max Coupling Flux (W/m^2) & $8.573 \mathrm{E}+05$ & $9.238 \mathrm{E}+05$ & $-7.200 \%$ \\
Min Coupling Flux (W/m^2) & $8.573 \mathrm{E}+05$ & $6.915 \mathrm{E}+05$ & $23.970 \%$ \\
Max Metal/Oxide Interface Temp (K) & 629.406 & 628.844 & $0.089 \%$ \\
Max Oxide Thickness (microns) & 14.1472 & 13.8592 & $2.078 \%$ \\
Total Oxide Mass (kg) & $5.607 \mathrm{E}-03$ & $5.588 \mathrm{E}-03$ & $0.338 \%$ \\
Max Clad Temperature (K) & 673.605 & 673.02 & $0.087 \%$ \\
Serial Run Time (s) & 5 & 73 & \\
\hline
\end{tabular}

Table 17. 3D vs. 1D model comparison at $160 \%$ power for 500 days.

\begin{tabular}{llll}
\hline & $1 \mathrm{D}$ & $3 \mathrm{D}$ & $\%$ Difference \\
\hline Max Surface Temperature (K) & 623.776 & 623.776 & \\
Min Surface Temperature (K) & 585.799 & 585.799 & \\
Max Crud Thickness (m) & $8.7997 \mathrm{E}-05$ & $8.7969 \mathrm{E}-05$ & $0.032 \%$ \\
Total Crud Mass (kg) & $2.1098 \mathrm{E}-03$ & $2.1073 \mathrm{E}-03$ & $0.120 \%$ \\
Total Boron Mass (kg) & $3.7605 \mathrm{E}-06$ & $3.7590 \mathrm{E}-06$ & $0.039 \%$ \\
Max Crud/Clad Interface Temp (K) & 623.917 & 623.914 & $0.000 \%$ \\
Max Coupling Flux (W/m²) & $9.797 \mathrm{E}+05$ & $1.035 \mathrm{E}+06$ & $-5.385 \%$ \\
Min Coupling Flux (W/m^2) & $9.797 \mathrm{E}+05$ & $8.327 \mathrm{E}+05$ & $17.658 \%$ \\
Max Metal/Oxide Interface Temp (K) & 634.706 & 634.161 & $0.086 \%$ \\
Max Oxide Thickness (microns) & 16.4889 & 16.3755 & $0.692 \%$ \\
Total Oxide Mass (kg) & $6.870 \mathrm{E}-03$ & $6.845 \mathrm{E}-03$ & $0.366 \%$ \\
Max Clad Temperature (K) & 685.111 & 684.154 & $0.140 \%$ \\
Serial Run Time (s) & 5 & 75 & \\
\hline
\end{tabular}


Table 18. 3D vs. $1 \mathrm{D}$ model comparison at $180 \%$ power for 500 days.

\begin{tabular}{llll}
\hline & 1D & 3D & \% Difference \\
\hline Max Surface Temperature (K) & 627.514 & 627.514 & \\
Min Surface Temperature (K) & 588.285 & 588.285 & \\
Max Crud Thickness (m) & $1.4872 \mathrm{E}-04$ & $1.4866 \mathrm{E}-04$ & $0.044 \%$ \\
Total Crud Mass (kg) & $3.5636 \mathrm{E}-03$ & $3.5618 \mathrm{E}-03$ & $0.049 \%$ \\
Total Boron Mass (kg) & $2.4667 \mathrm{E}-05$ & $2.4660 \mathrm{E}-05$ & $0.030 \%$ \\
Max Crud/Clad Interface Temp (K) & 627.789 & 627.78 & $0.001 \%$ \\
Max Coupling Flux (W/m^2) & $1.102 \mathrm{E}+06$ & $1.159 \mathrm{E}+06$ & $-4.897 \%$ \\
Min Coupling Flux (W/m^2) & $1.102 \mathrm{E}+06$ & $1.014 \mathrm{E}+06$ & $8.703 \%$ \\
Max Metal/Oxide Interface Temp (K) & 642.973 & 642.05 & $0.144 \%$ \\
Max Oxide Thickness (microns) & 20.6193 & 20.3844 & $1.152 \%$ \\
Total Oxide Mass (kg) & $7.949 \mathrm{E}-03$ & $7.918 \mathrm{E}-03$ & $0.394 \%$ \\
Max Clad Temperature (K) & 699.466 & 697.927 & $0.221 \%$ \\
Serial Run Time (s) & 5 & 79 & \\
\hline
\end{tabular}

\subsection{Model Comparison for Thick Oxide Layers}

The difference between 1D and 3D models was assessed for thicker oxide layers by using the fast flux value of $6.0 \mathrm{e} 17$ as used in numerous calculations in the IAEA report [1]. This larger value of fast flux increases the irradiation enhancement factor in Eq (14) which subsequently increases the post-transition growth rate of the oxide resulting in a thicker overall crud layer. The calculations of the previous section were repeated at power levels of $180 \%$ and $160 \%$ using the larger fast flux value with the results given in Table 19 and Table 20. These results show that as oxidation increases, the geometric effects included in the 3D model become more apparent resulting in cooler clad temperatures and reduced oxidation compared to the $1 \mathrm{D}$ model which does not allow heat to flow around thick thermal resistors. In addition, similar variations in $q_{\text {surf }}^{\prime \prime}$ are noted with the variation between $1 \mathrm{D}$ and $3 \mathrm{D}$ even larger for thick oxide layers. The crud results, however, were effectively the same between the thick and thin oxide cases, indicating that the crud model used in this work is predominately affected by the surface conditions generated by the CFD calculation. 
Table 19. 3D vs. 1D model comparison at $180 \%$ power for 500 days with fast flux parameter of $6.0 \mathrm{e} 17 \mathrm{n} / \mathrm{cm}^{\wedge} 2$-s as defined by IAEA report.

\begin{tabular}{llll}
\hline & $1 \mathrm{D}$ & 3D & \% Difference \\
\hline Max Surface Temperature (K) & 627.514 & 627.514 & \\
Min Surface Temperature (K) & 588.285 & 588.285 & \\
Max Crud Thickness (m) & $1.4872 \mathrm{E}-04$ & $1.4864 \mathrm{E}-04$ & $0.059 \%$ \\
Total Crud Mass (kg) & $3.5631 \mathrm{E}-03$ & $3.5599 \mathrm{E}-03$ & $0.088 \%$ \\
Total Boron Mass (kg) & $2.4667 \mathrm{E}-05$ & $2.4659 \mathrm{E}-05$ & $0.032 \%$ \\
Max Crud/Clad Interface Temp (K) & 627.789 & 627.773 & $0.003 \%$ \\
Max Coupling Flux (W/m^2) & $1.102 \mathrm{E}+06$ & $1.183 \mathrm{E}+06$ & $-6.806 \%$ \\
Min Coupling Flux (W/m²) & $1.102 \mathrm{E}+06$ & $9.932 \mathrm{E}+05$ & $10.971 \%$ \\
Max Metal/Oxide Interface Temp (K) & 683.042 & 675.02 & $1.188 \%$ \\
Max Oxide Thickness (microns) & 74.6037 & 69.535 & $7.289 \%$ \\
Total Oxide Mass (kg) & $2.081 \mathrm{E}-02$ & $2.067 \mathrm{E}-02$ & $0.717 \%$ \\
Max Clad Temperature (K) & 736.74 & 728.892 & $1.077 \%$ \\
Serial Run Time (s) & 5 & 305 & \\
\hline
\end{tabular}

Table 20. 3D vs. 1D model comparison at $160 \%$ power for 500 days with fast flux parameter of $6.0 \mathrm{e} 17 \mathrm{n} / \mathrm{cm}^{\wedge} 2$-s as defined by IAEA report.

\begin{tabular}{llll}
\hline & 1D & 3D & \% Difference \\
\hline Max Surface Temperature (K) & 623.776 & 623.776 & \\
Min Surface Temperature (K) & 585.799 & 585.799 & \\
Max Crud Thickness (m) & $8.7997 \mathrm{E}-05$ & $8.7963 \mathrm{E}-05$ & $0.039 \%$ \\
Total Crud Mass $(\mathrm{kg})$ & $2.1100 \mathrm{E}-03$ & $2.1071 \mathrm{E}-03$ & $0.139 \%$ \\
Total Boron Mass $(\mathrm{kg})$ & $3.7606 \mathrm{E}-06$ & $3.7590 \mathrm{E}-06$ & $0.043 \%$ \\
Max Crud/Clad Interface Temp (K) & 623.917 & 623.912 & $0.001 \%$ \\
Max Coupling Flux (W/m^2) & $9.797 \mathrm{E}+05$ & $1.047 \mathrm{E}+06$ & $-6.403 \%$ \\
Min Coupling Flux (W/m^2) & $9.797 \mathrm{E}+05$ & $8.170 \mathrm{E}+05$ & $19.915 \%$ \\
Max Metal/Oxide Interface Temp (K) & 654.524 & 652.119 & $0.369 \%$ \\
Max Oxide Thickness (microns) & 46.6294 & 45.5138 & $2.451 \%$ \\
Total Oxide Mass (kg) & $1.654 \mathrm{E}-02$ & $1.643 \mathrm{E}-02$ & $0.627 \%$ \\
Max Clad Temperature (K) & 703.547 & 700.982 & $0.366 \%$ \\
Serial Run Time (s) & 5 & 188 & \\
\hline
\end{tabular}

\subsection{Algorithmic Assessment Summary}

In summary:

- For a fixed set of boundary conditions in a given conjugate heat transfer iteration (i.e. a given $T_{\text {surf }}$ and $q_{\phi}^{\prime \prime}$ ), a 1D model is sufficient for computing crud growth, boron deposition, and oxidation at CFD length scales when the oxide layer is relatively thin.

- Discrepancies between the 1D and 3D models increase as oxidation increases due to increased geometric effects captured by the 3D model. It is not clear if these effects are substantial enough to warrant using the $3 \mathrm{D}$ vs. the less expensive $1 \mathrm{D}$ model. 
- Significant variation is noted between the $1 \mathrm{D}$ and $3 \mathrm{D}$ models in the computed $q_{\text {surf }}^{\prime \prime}$ due to the same geometric effects.

- If coupling between the clad model and the flow model is strong, then the large observed variations in $q_{\text {surf }}^{\prime \prime}$ could significantly affect the result of the conjugate heat transfer calculation.

- Using the 1D and 3D clad models in a full conjugate heat transfer simulation with STAR$\mathrm{CCM}+$ will determine which is needed for CILC modeling assuming the fuel and gap is not to be modeled.

- Spatial variation in the heat flux on the inner surface of the clad model, $q_{\phi}$, and its effects on the models will be assessed by including heat transfer in the fuel and gap.

- A scheme has been established to use DTK to couple STAR-CCM+ with a clad model consisting of crud, corrosion, and thermal models. This scheme can be used to add other crud and corrosion models for further algorithmic analysis (e.g. 3D MAMBA).

- The new model can serve as an alternative to performing heat transfer in the rod in STAR$\mathrm{CCM}+$, potentially reducing overall run times and simplifying the modeling process by requiring only fluid flow to be modeled. 


\section{CONCLUSION}

In this milestone we have developed new capabilities in the Cicada package to move towards CFD-informed subchannel modeling of CIPS and CILC phenomena. The new tools allow for the direct comparison of STAR-CCM+ and CTF results to drive further model development. In addition, a new HDF5 feature and supporting set of post-processing tools allows for further analysis. We also developed new infrastructure in Cicada to allow for the coupling of MAMBA 3D using the DTK library. With these new tools we have begun to assess the coupling of various physics needed by high-fidelity CILC modeling to better understand where high fidelity results are needed and where approximations are sufficient. Initial analysis of 3D vs 1D models indicates that it may be possible to use simpler and more efficient 1D models in many cases. However, this result should be further investigated in more sophisticated simulations.

As future work we will continue to develop CFD-informed subchannel capability as it relates to CIPS and CILC as part of the Cicada package. In addition, we plan to incorporate coupling to MAMBA 3D to assess its affects on the coupling algorithm presented in this work. Finally, we will continue to assess potential approximations that can be used to make the modeling of CILC with STAR-CCM+ more computationally efficient. 


\section{References}

[1] International Atomic Energy Agency. Waterside corrosion of zirconium alloys in nuclear power plants. IAEA-TECDOC-996. 1998.

[2] B. Nadiga et al. Surface Chemistry Capability in Hyrda-TH (L3 Milestone THM.CFD.P9.11). LA-UR 14-27156. 2014.

[3] V. Petrov et al. "Impact of 3D Spatial Variations in Fluid Flow on the Predicition of CRUD deposition in a 4X4 PWR Sub-assembly". In: The 15th International Topical Meeting on Nuclear Reactor Thermalhydraulics, NURETH-15, Pisa, Italy, May 12-17, 2013 (2013).

[4] V. Petrov et al. Prediction of CRUD Deposition on PWR Fuel Using a State-of-the-Art CFD-Based Multi-physics Computational Tool. 2014.

[5] R. Salko et al. Milestone L3:PHI.CTF.P10.02 Coupling of Subchannel T/H (CTF) and CRUD Chemistry (MAMBA). 2015.

[6] S. Slattery. "Mesh-free data transfer algorithms for multiphysics problems: Conservation, accuracy, and parallelism". In: Journal of Computational Physics 207 (2016), pp. 164-188.

[7] SR Slattery, PPH Wilson, and RP Pawlowski. "The Data Transfer Kit: A Geometric Rendezvous-Based Tool for Multiphysics Data Transfer". In: International Conference on Mathematics $\& 3$ Computational Methods Applied to Nuclear Science $\mathcal{E}$ Engineering (MEC 2013) (CD-ROM). May 2013.

[8] D. Walter et al. "Proof-of-principle of high-fidelity coupled CRUD deposition and cycle depletion simulation". In: Annals of Nuclear Energy - In Press (2015).

[9] D. Walter et al. "Sensitivity of CRUD Deposition Predictions: Feedback Effects of Thermal Hydraulics and Lattice Depletion". In: PHYSOR 2016 - submitted (2016).

[10] D. Walter et al. "Sensitivity of CRUD Deposition Predictions: Fixed Thermal Hydraulic Boundary Conditions". In: PHYSOR 2016 - submitted (2016). 